\title{
EFECTO DE LA DENSIDAD DE SIEMBRA Y VOLUMEN DE SUSTRATO SOBRE PARÁMETROS DE RIEGO Y RENDIMIENTO DE CHILE DULCE 'DULCITICO', EN HIDROPONÍA BAJO INVERNADERO
}

\author{
Freddy Soto-Bravo ${ }^{1 *}$, Elvis Ariel Araya-Cubero², Carlos Echandi-Gurdian ${ }^{3}$ \\ Palabras clave: Manejo del riego; humedad del sustrato; densidad de siembra; volumen de sustrato. \\ Keywords: Irrigation management; substrate water content; planting density; substrate volume.
}

\section{RESUMEN}

La investigación se realizó de abril a setiembre de 2016, en la Estación Experimental Agrícola Fabio Baudrit Moreno de la Universidad de Costa Rica, Alajuela, Costa Rica, con el híbrido costarricense de chile dulce 'Dulcitico', en sistema hidropónico bajo invernadero. Se evaluó el efecto de 2 densidades de siembra (1,4 y 2,4 plantas. $\left.\mathrm{m}^{-2}\right)$ y 3 volúmenes de sustrato $(7$, 14 y 21 L.planta $^{-1}$ ) sobre el rendimiento, contenido de agua total disponible (ATD) en el sustrato, drenaje, evapotranspiración del cultivo $\left(\mathrm{ET}_{\mathrm{c}}\right) \mathrm{y}$ eficiencia de uso de agua (EUA). Se utilizó un diseño de 3 bloques completos al azar con arreglo bifactorial de tratamientos $2 \times 3$. La ET $\mathrm{c}$ se obtuvo mediante balance de agua en el sustrato y la EUA como el cociente entre rendimiento y la $\mathrm{ET}_{\mathrm{c}}$. Para todas las variables de respuesta, no se observaron diferencias entre ambas densidades de siembra, conforme se incrementó el volumen de sustrato desde 7 hasta $21 \mathrm{~L}(\mathrm{p}<0,05)$. Ambos factores afectaron $(\mathrm{p}<0,05)$ de forma independiente a todas las variables; excepto el ATD y el

\footnotetext{
* Autor para correspondencia. Correo electrónico: freddy.sotobravo@ucr.ac.cr

1 Universidad de Costa Rica, Estación Experimental Agrícola Fabio Baudrit Moreno, Facultad de Ciencias Agroalimentarias, Costa Rica. (iD) 0000-0003-1959-9597.
}

\begin{tabular}{|c|c|}
\hline & ABSTRACT \\
\hline & 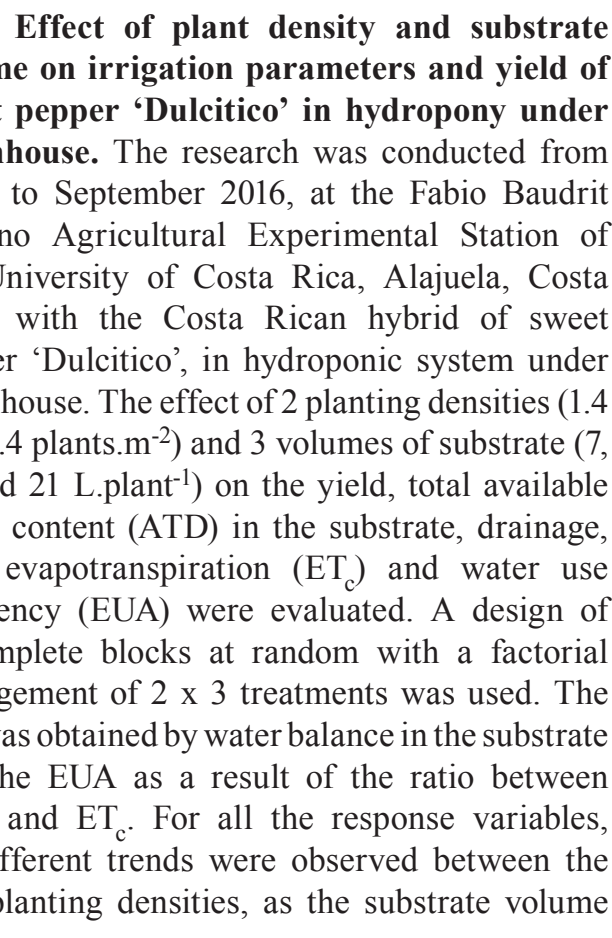 \\
\hline & $\begin{array}{l}\text { Universidad de Costa Rica, Estación Experimental } \\
\text { Agrícola Fabio Baudrit Moreno, Facultad de } \\
\text { Ciencias Agroalimentarias, Costa Rica. } \\
\text { (DD } 0000-0002-7936-2666 \text {. }\end{array}$ \\
\hline 3 & $\begin{array}{l}\text { Universidad de Costa Rica, Estación Experimental } \\
\text { Agrícola Fabio Baudrit Moreno, Facultad de } \\
\text { Ciencias Agroalimentarias, Costa Rica. } \\
\text { (D) } 0000-0001-6653-7474 \text {. }\end{array}$ \\
\hline
\end{tabular}


rendimiento, que únicamente fueron afectados $(\mathrm{p}<0,05)$ por el volumen de sustrato y la densidad de plantas, respectivamente. En todos los casos, el efecto de mayor magnitud fue debido al factor densidad. El volumen de sustrato no afectó el rendimiento, sin embargo, contribuyó a mejorar la EUA al reducir los volúmenes de riego, de $\mathrm{ET}_{\mathrm{c}}$ y de drenaje. A mayor densidad de siembra incrementó el rendimiento $\left(\mathrm{kg} . \mathrm{m}^{-2}\right)$, pero redujo la EUA, debido a un mayor consumo de agua $\left(\mathrm{ET}_{\mathrm{c}}\right)$, y mayores volúmenes de riego, de ATD y de drenaje. La mayor EUA se obtuvo a 1,4 plantas. $\mathrm{m}^{-2}$ y $7 \mathrm{~L}$ de sustrato.planta ${ }^{-1}$, por una reducción de $13 \%$ en la $\mathrm{ET}_{\mathrm{c}}$ respecto al cultivo con $21 \mathrm{~L}$ de sustrato.planta ${ }^{-1}$ y 2,4 plantas. $\mathrm{m}^{-2}$.

\section{INTRODUCCIÓN}

En Costa Rica para el 2014, se cultivaron aproximadamente 1085,5 ha de chile dulce (INEC 2015) de las cuales, según Rojas y Paniagua (2015), 158 ha fueron bajo invernadero. En este cultivo existe una fuerte dependencia de semilla importada con un alto costo. La Universidad de Costa Rica desarrolló el primer híbrido nacional de chile dulce "Dulcitico" adaptado a las condiciones agroecológicas del país. Sin embargo, es necesario desarrollar tecnología adaptada para alcanzar su máximo potencial productivo.

A nivel global, los recursos hídricos son limitantes, ya que representan tan solo el 1,5\% de los recursos de agua total del planeta, del cual el sector agropecuario es el principal consumidor de agua (69\%). El crecimiento demográfico mundial, proyectado a 9000 millones de habitantes en el 2050 (Tilman et al. 2002), supone un incremento sustancial en la demanda de alimentos y asimismo de agua. Se estima que para el 2050 was increased from 7 to $21 \mathrm{~L}(\mathrm{p}<0.05)$. Both factors affected $(\mathrm{p}<0.05)$ independently all the variables; except the ATD and yield, which were only affected $(p<0.05)$ by the volume of substrate and plant density, respectively. In all cases, the effect of greater magnitude was due to the density factor. The volume of substrate did not affect the yield, however, it contributed to improve the EUA by reducing the volumes of irrigation, $\mathrm{ET}_{\mathrm{c}}$ and drainage. Higher planting density increased yield $\left(\mathrm{kg} . \mathrm{m}^{-2}\right)$, but reduced the EUA due to higher water consumption $\left(\mathrm{ET}_{\mathrm{c}}\right.$ ) and higher volumes of irrigation, ATD and drainage. The highest EUA was obtained at 1.4 plants. $\mathrm{m}^{-2}$ and $7 \mathrm{~L}$ of substrate.plant ${ }^{-1}$, due to a reduction of $13 \%$ in the $\mathrm{ET}_{\mathrm{c}}$ with respect to the crop with $21 \mathrm{~L}$ of substrate.plant ${ }^{-1}$ and 2.4 plants. $\mathrm{m}^{-2}$.

dicho requirimiento se incrementará en un $66 \%$ para alimentos vegetales y un $76 \%$ para alimentos pecuarios (WWAP 2016). La escasez de agua dulce para riego podría duplicar los efectos perjudiciales del cambio climático en la agricultura, debido a la reducción de los rendimientos en los principales cultivos como maíz, soja, trigo y arroz (Elliott et al. 2014).

La evolución de agricultura de aproximación a agricultura de precisión, está contribuyendo al uso racional de los recursos de producción. Aunque existe la tecnología, esta puede ser mejor aprovechada para realizar el uso eficiente de agua de riego. En ese sentido, la hidroponía contribuye con la eficiencia de uso de agua, de los fertilizantes y del espacio, reduce enfermedades de suelo y aumenta la calidad y los rendimientos (Jara 2016). La producción de cultivos, sin suelo en invernadero, reduce los efectos de climas extremos, permite producir hortalizas durante todo el año e incrementa la calidad y el rendimiento. Además, reduce la incidencia de plagas 
y enfermedades, lo cual disminuye el uso de pesticidas, lo que genera productos más sanos para la persona consumidora y menor contaminación ambiental (Rojas y Paniagua 2015).

Las densidades de siembra a campo abierto $\mathrm{y}$ en invernadero, utilizadas en Costa Rica para chile, dulce son variables y fluctúan entre 1,4 hasta 6,0 plantas $\mathrm{m}^{-2}$. Silva et al. (2010) evaluaron 4 densidades de siembra en chile dulce 2, 2,5, 3,3 y 5 plantas. $\mathrm{m}^{-2}$ y determinaron que el efecto de la densidad de plantación, sobre el crecimiento y producción del cultivo, está determinado únicamente por la distancia entre plantas sin efecto de la distancia entre hileras.

Existe amplia investigación sobre el efecto de déficit de riego en chile dulce (Koksal et al. 2017, Hakk1 and Y1ldırım 2015, Guang-Cheng et al. 2010, Kirda et al. 2007) y el uso de injertos (López-Marín et al. 2017), sobre la EUA. Sin embargo, existe un vacío de información acerca de otros factores de manejo como la densidad de siembra y el volumen de sustrato sobre la EUA en cultivos en invernadero como el híbrido nacional 'Dulcitico', el cual se produce bajo condiciones tropicales.

La correcta estimación de la evapotranspiración del cultivo $\left(\mathrm{ET}_{\mathrm{c}}\right)$ permite mejorar la EUA y aumentar la calidad y el rendimiento (Pérez y Castellvì 2002). Aplicaciones excesivas de agua, debido a la falta de datos locales de $\mathrm{ET}_{\mathrm{c}}$, disminuyen la EUA. Actualmente, en Costa Rica no hay estudios acerca de la $\mathrm{ET}_{\mathrm{c}}$, porcentajes de drenaje y EUA en cultivos en cultivos en invernadero. En España, estudios realizados por Fernández et al. (2005) en chile dulce bajo invernadero, reportaron valores de $\mathrm{ET}_{\mathrm{c}}$ total de 137-362 L.m ${ }^{-2}$, mientras que Karam et al. (2009) en chile Bell pepper determinaron rangos de $\mathrm{ET}_{\mathrm{c}}$ entre 275 - 478 L.m${ }^{2}$. No obstante, la utilización de estos datos para efectos de manejo puede ser contraproducente, ya que la $\mathrm{ET}_{\mathrm{c}}$ varía con el genotipo, el clima y el manejo agronómico del cultivo. Únicamente se encontró un estudio local por Soto (2018), que reportó que el umbral de humedad en el cultivo de chile dulce en fibra de coco bajo invernadero, antes de afectar fisiológicamente la planta, fue aproximadamente $38 \%$ de humedad a una succión $5 \mathrm{kPa}$.

El objetivo de este estudio fue evaluar el efecto de la densidad de siembra y el volumen de sustrato sobre el rendimiento, la evapotranspiración del cultivo $\left(\mathrm{ET}_{\mathrm{c}}\right)$, el drenaje, el agua total disponible (ATD) en el sustrato y la eficiencia física de uso de agua (EUA) en el híbrido $\mathrm{F}_{1}$ costarricense "Dulcitico" de chile dulce hidropónico bajo invernadero.

\section{MATERIALES Y MÉTODOS}

Sitio experimental. El estudio se realizó del 22 de abril al 28 de agosto del 2016, en la Estación Experimental Agrícola Fabio Baudrit Moreno, ubicada en San José de Alajuela, Costa Rica ( $10^{\circ} 00^{\prime} 24^{\prime} \mathrm{N}$ y $\left.84^{\circ} 15^{\prime} 57^{\prime} \mathrm{W}\right)$, a una altitud de $840 \mathrm{msnm}$, con valores promedios de precipitación anual, temperatura y humedad relativa de $1940 \mathrm{~mm}, 22^{\circ} \mathrm{C}$ y $78 \%$, respectivamente.

Se utilizó un invernadero multitúnel de hierro galvanizado con cubierta de polietileno $(200 \mu \mathrm{m})$ y malla anti-insectos (43 mesh) en las paredes, formado por 4 módulos de $9,75 \mathrm{~m}$ de ancho y $50 \mathrm{~m}$ de largo $\left(1440 \mathrm{~m}^{2}\right)$, alturas de $6 \mathrm{~m}$ al centro del túnel y $4 \mathrm{~m}$ a la canoa. El sistema de ventilación fue pasivo, combinado con el funcionamiento automatizado de ventanas cenitales según la velocidad de viento y lluvia.

Durante el experimento, se monitoreó la radiación solar global, la temperatura y la humedad del aire en el interior del invernadero, que fueron almacenados en un registro de datos (Figura 1). 


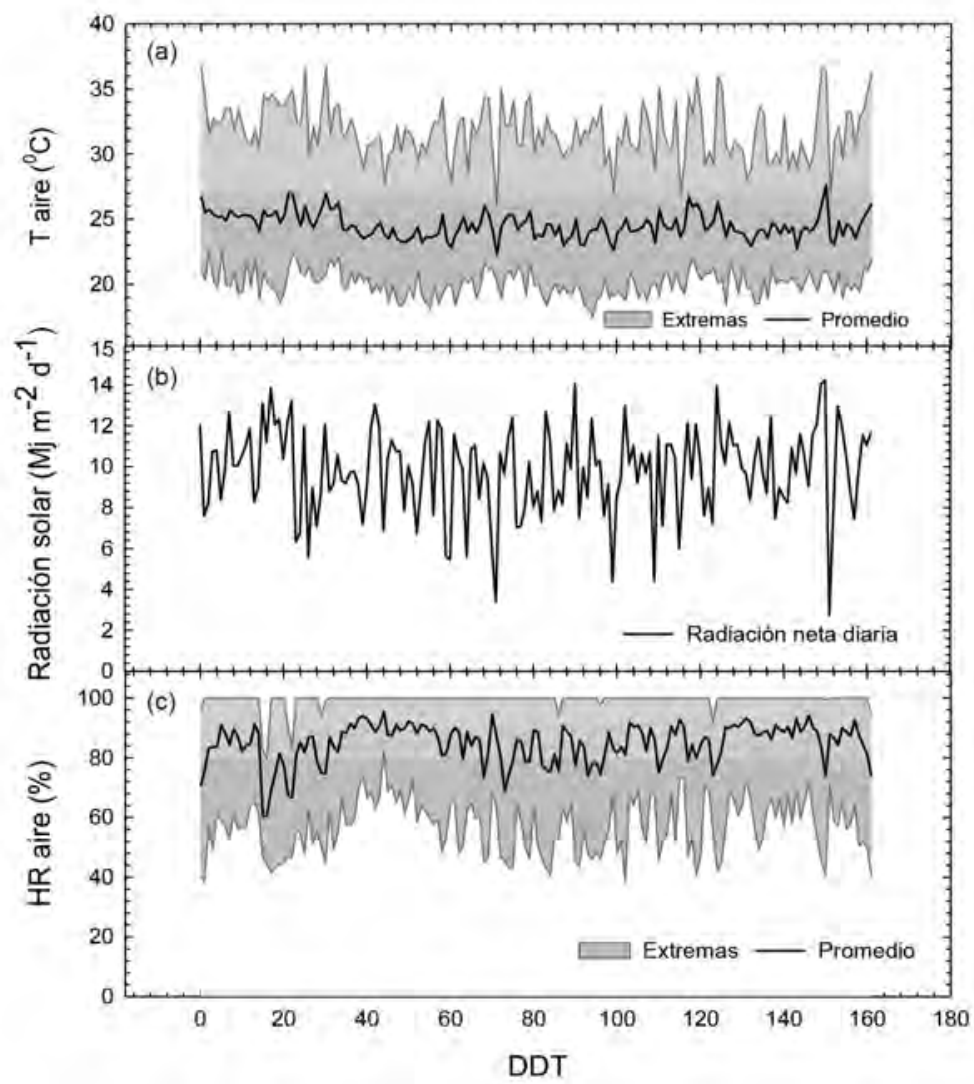

Figura 1 Condiciones de invernadero durante el ciclo de cultivo de chile dulce "Dulcitico", Alajuela, Costa Rica.
(a) Valores máximo promedio y mínimo de temperatura.
(b) Humedad relativa.
(c) Aire e integral de radiación solar global.

Se utilizó el híbrido $F_{1}$ "Dulcitico”, desarrollado en Costa Rica, que se caracteriza por iniciar la cosecha entre 90 y 110 días después del trasplante, de crecimiento indeterminado, fruto de forma cónica, color verde-rojo, altos grados brix y rendimiento de hasta 9,55 kg.m ${ }^{-2}$ (Echandi 2012). Para el trasplante se utilizaron plántulas de 37 días de edad con 6 hojas verdaderas, obtenidas en bandejas plásticas de 98 celdas con turba (peat moss) como medio de cultivo.

El sustrato utilizado en el experimento fue fibra de coco de textura media en bloques comprimidos de $100 \times 15 \times 3 \mathrm{~cm}$, que fueron hidratados, descomprimidos y determinadas sus características físicas (Tabla 1). La porosidad total $\left(\% \mathrm{P}_{t}\right)$ se obtuvo a partir de la densidad aparente $\left(\mathrm{d}_{\mathrm{ap}}\right)$ y la densidad real $\left(\mathrm{d}_{\mathrm{r}}\right)$, mediante la ecuación Pt $=\left(1-\mathrm{d}_{\mathrm{ap}} / \mathrm{d}_{\mathrm{r}}\right) * 100$. La $\mathrm{d}_{\mathrm{ap}}\left(\mathrm{g} \mathrm{cm}^{-3}\right)$ se determinó a partir del cociente entre el peso seco a $105^{\circ} \mathrm{C}$ y el volumen de sustrato, mientras que la $\mathrm{d}_{\mathrm{r}}\left(\mathrm{g} \mathrm{cm}^{-3}\right)$ se determinó por el método del picnómetro. El contenido de partículas sólidas (\%) se obtuvo como la diferencia entre $100-\mathrm{P}_{\mathrm{t}}$. 
Tabla 1. Características físicas de la fibra de coco utilizada como sustrato para el cultivo de chile dulce hidropónico bajo invernadero.

\begin{tabular}{lcc}
\hline Características físicas & Fibra de coco & \% óptimo \\
\hline Partículas $<1 \mathrm{~mm}=$ índice de grosor (\%) & 83,0 & -- \\
Partículas entre 1 y 4 mm (\%) & 58,00 & -- \\
Partículas > $4 \mathrm{~mm}(\%)$ & 25,00 & -- \\
Densidad aparente (gr.cm $\left.{ }^{-3}\right)$ & 0,075 & -- \\
Densidad real $\left(\mathrm{gr.cm}^{-3}\right)$ & 0,650 & $>85 \%$ \\
Porosidad total (\%) & 88,5 & $<15 \%$ \\
Partículas sólidas (\%) & 11,5 & - \\
\hline
\end{tabular}

Los componentes de agua y aire en la fibra de coco se determinaron según la norma europea UNE-EN-13041 (2007). Para ello, se determinó el porcentaje de humedad volumétrica $(\theta)$ en muestras sometidas a potenciales mátricos $\left(\psi_{\mathrm{m}}\right)$ de $1,2,5,5,0,7,5$ y $10 \mathrm{kPa}$, utilizando un equipo de caja de arena. Para cada $\psi_{\mathrm{m}}$ se utilizaron 3 repeticiones y se asumió que la $\theta$ a $1 \mathrm{kPa}$ y a $10 \mathrm{kPa}$ correspondieron a la capacidad de contenedor $\left(\theta_{\mathrm{CC}}\right)$ y al punto de marchitez permanente $\left(\theta_{\mathrm{PMP}}\right)$, respectivamente. La capacidad de aireación (\%) del sustrato en cada $\psi_{\mathrm{m}}$ se obtuvo como la diferencia entre la $\mathrm{P}_{\mathrm{t}} \mathrm{y}$ el porcentaje de $\theta$ respectivo. El porcentaje de agua total disponible (\%ATD) en el sustrato $\left(\theta_{\mathrm{CC}}-\theta_{\mathrm{PMP}}\right)$ incluyó los porcentajes de agua fácilmente disponible (\%AFD) y agua de reserva (\%AR). El \%AFD se obtuvo a partir de la diferencia entre $\theta_{\mathrm{CC}}$ y la humedad a $5 \mathrm{kPa}\left(\theta_{5 \mathrm{kPa}}\right)$, mientras que el $\% \mathrm{AR}$ correspondió a la diferencia entre $\theta_{5 \mathrm{kPa}}$ y $\theta_{\mathrm{PMP}}$. La fibra de coco utilizada presentó una $\theta_{\mathrm{CC}} \mathrm{de}$ $57,4 \%(1 \mathrm{kPa})$ y una $\theta_{\text {PMP }}$ de $30,86 \%(10 \mathrm{kPa})$, para un 26,54\% de ATD. El AFD fue de 22,23\%, almacenada entre $\theta_{\mathrm{CC}}$ y $\theta_{5 \mathrm{kPa}}$ (Figura 2).

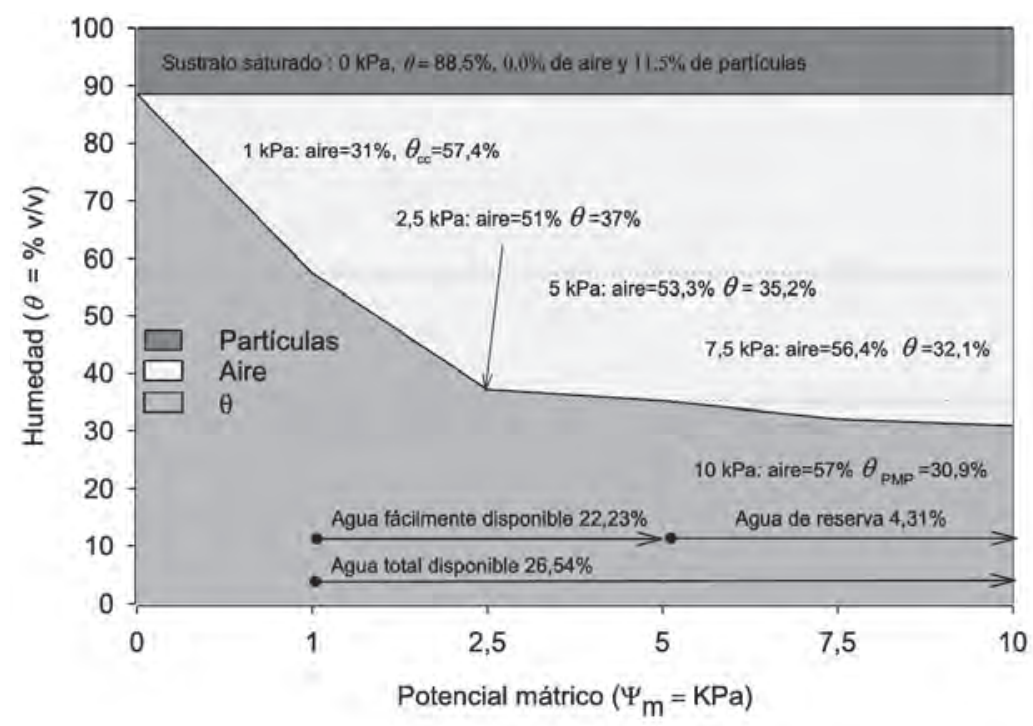

Figura 2 Curva característica de liberación de aire y desorción de agua, agua fácilmente disponible, agua de reserva y agua total disponible en la fibra de coco, respectivamente. 
Tratamientos. Se evaluaron 6 tratamientos que combinaban 2 densidades de siembra (D) y 3 volúmenes de sustrato por planta (V). Las densidades usadas fueron 1,4 plantas. $\mathrm{m}^{-2}(0,45 \mathrm{~m}$ x $1,55 \mathrm{~m})$ y 2,4 plantas. $\mathrm{m}^{-2}$ $(0,27 \mathrm{~cm} \mathrm{x} 1,55 \mathrm{~cm})$; mientras que los volúmenes de sustrato fueron $7 \mathrm{~L}, 14 \mathrm{~L}$ y $21 \mathrm{~L}^{\text {. }}$ planta $^{-1}$ (Tabla 2).

Tabla 2. Descripción de tratamientos utilizados según densidad de siembra y volumen de sustrato.

\begin{tabular}{cccccc}
\hline \multirow{2}{*}{ Código } & \multicolumn{2}{c}{ Densidad de plantas } & Volumen de sustrato & \multicolumn{2}{c}{ Distancia (m) } \\
& $\mathrm{Ha}$ & $\mathrm{m}^{2}$ & por $(\mathrm{L})$ & Hileras & Plantas \\
\hline $\mathrm{D}_{1,4} \mathrm{~V}_{7}$ & 1,4 & 7 & 1,55 & 0,45 \\
$\mathrm{D}_{1,4} \mathrm{~V}_{14}$ & 14000 & 1,4 & 14 & 1,55 & 0,45 \\
$\mathrm{D}_{1,4} \mathrm{~V}_{21}$ & & 1,4 & 21 & 1,55 & 0,45 \\
$\mathrm{D}_{2,4} \mathrm{~V}_{7}$ & & 2,4 & 7 & 1,55 & 0,27 \\
$\mathrm{D}_{2,4} \mathrm{~V}_{14}$ & 24000 & 2,4 & 14 & 1,55 & 0,27 \\
$\mathrm{D}_{2,4} \mathrm{~V}_{21}$ & & 2,4 & 21 & 1,55 & 0,27 \\
\hline
\end{tabular}

La fibra de coco hidratada y descomprimida se colocó en bolsas plásticas de color blanco de 8 milésimas de espesor. De acuerdo con el volumen de sustrato por tratamiento, el ancho y el alto de la bolsa llena de sustrato mantuvo una relación constante de $1: 1$, con $20 \mathrm{~cm}$ x $20 \mathrm{~cm}$ para $7 \mathrm{~L}, 25 \mathrm{~cm}$ x $25 \mathrm{~cm}$ para $14 \mathrm{~L}$ y $28 \mathrm{~cm}$ x $28 \mathrm{~cm}$ para $21 \mathrm{~L}$. Las bolsas con el sustrato se distribuyeron en el área experimental según tratamiento, y seguidamente, se trasplantó una plántula de chile dulce en cada bolsa.

Manejo del experimento. El sistema de riego consistió de un reservorio de agua de 10000 litros, una bomba eléctrica (1 HP), tubería principal de polietileno $(25 \mathrm{~mm})$, tubería de polietileno $(19 \mathrm{~mm})$ para cada hilera de cultivo con un gotero (3 L.h ${ }^{-1}$ ) antidrenante y autocompensado para cada planta/bolsa. Cada gotero constaba de un difusor de 4 salidas con microtubos (5,5 $\mathrm{mm})$ de $60 \mathrm{~cm}$ de largo con una estaca de anclaje al final, colocadas alrededor de la base de cada planta. Para reducir la superficie expuesta a la evaporación de agua, el sustrato se cubrió con el sobrante de plástico de la misma bolsa, dejando un pequeño espacio $(3 \mathrm{~cm})$ alrededor del tallo en la base de la planta. La presión del sistema $(103,4$ $\mathrm{kPa})$ y el control del caudal fueron regulados, a través de un tubo con llave de paso, que retornaba parte del agua impulsada por la bomba al tanque de almacenamiento.

La nutrición mineral se inició un día después del trasplante, con una solución nutritiva según etapa del cultivo (Tabla 3). El pH de la solución nutritiva fue ajustado a 5,7 con ácido nítrico $\left(56 \% \mathrm{v} / \mathrm{v}, 167\right.$ g.L $\left.\mathrm{L}^{-1}\right)$ según la concentración de bicarbonatos (77 mg. $\left.\mathrm{L}^{-1}\right)$ en el agua de riego. Las concentraciones de nutrientes en el agua de riego fueron consideradas en los aportes a la solución nutritiva. Dichas concentraciones fueron 6,2 de $\mathrm{N}^{-\mathrm{NO}_{3}}{ }^{-}, 0,3$ de $\mathrm{P}, 3,7$ de $\mathrm{K}, 12,6$ de $\mathrm{Ca}, 6,2$ de $\mathrm{Mg}$ y 1,6 mg. $\mathrm{L}^{-1}$ de S, con un $\mathrm{pH}$ de 7,5 y una conductividad eléctrica (CE) de $0,2 \mathrm{mS} \cdot \mathrm{cm}^{-1}$. 


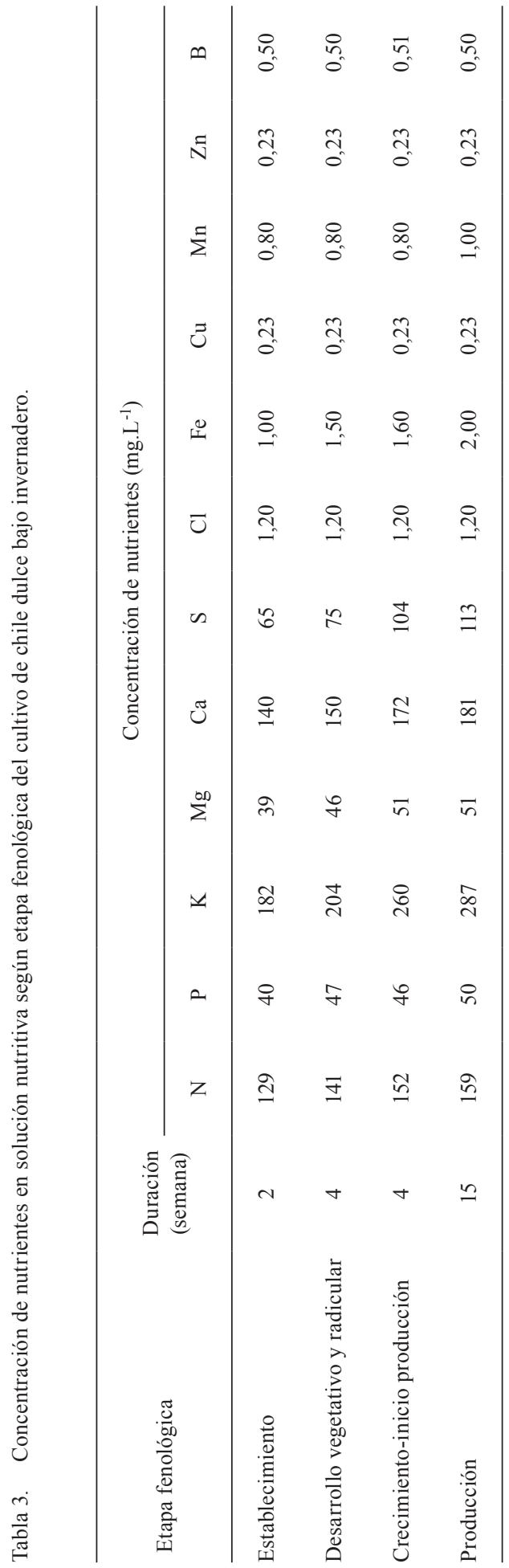

Agronomía Costarricense 44(1): 43-64. ISSN:0377-9424 / 2020 
En la preparación de la solución nutritiva, se utilizó fosfato monopotásico, nitrato de potasio, sulfato de potasio, sulfato de magnesio, nitrato de calcio, cloruro de calcio, ácido nítrico, elementos menores, ácido bórico, y quelatos de hierro y de manganeso (EDTA-Fe, EDTA-Mn). Para la protección del cultivo, previo al trasplante, se realizó una desinfección general del invernadero utilizando peróxido de hidrogeno $\left(\mathrm{H}_{2} \mathrm{O}_{2}\right)$. El manejo de los problemas fitosanitarios se realizó básicamente de forma preventiva mediante aplicaciones semanales de productos biológicos. Todas las semanas se monitoreaba la incidencia de insectos y patógenos, y si el umbral de plaga lo justificaba se realizaban aplicaciones de productos químicos específicos.

El tutorado de las plantas se realizó con mecate de nylon y anillos plásticos que se colocaron en la primera y segunda bifurcación de la planta. Posteriormente, todas las ramas emergentes se sujetaron con cuerda de nylon a un alambre metálico colocado horizontalmente a 2,5 $\mathrm{m}$ en la parte superior del cultivo. Todos los brotes vegetativos ubicados por debajo de la primera bifurcación de la planta fueron eliminados, mientras que por encima de este se permitió el libre crecimiento de tallos, y solamente, se realizó una deshoja sanitaria a los 105 días después del trasplante.

Diseño experimental. Los 6 tratamientos fueron distribuidos en un diseño de 3 bloques completos al azar, con un arreglo bifactorial 2 x 3 que combinaba 2 densidades de siembra y 3 volúmenes de sustrato por planta, para un total de 18 unidades experimentales (UE). En los tratamientos de baja densidad, la UE estaba conformada por 3 hileras de 9 plantas, para un total de 27 plantas; mientras que en los tratamientos de alta densidad estaban compuestos por 3 hileras de 13 plantas, para un total de 39 plantas. La unidad de muestreo constó de 7 plantas ubicadas en el centro de la hilera central de cada UE, utilizando como borde las 2 hileras externas y las plantas ubicadas en los extremos de la hilera central. Adicionalmente, se colocó una hilera extra contiguo a la pared externa del invernadero para mitigar el efecto de borde.

Variables de respuesta. Para cada tratamiento se determinó la evapotranspiración del cultivo ( $\mathrm{ET}_{\mathrm{c}}$ ) semanalmente, utilizando el método de balance de agua en el sistema, a partir de los volúmenes $\left({\left.\mathrm{L} . \mathrm{m}^{-2}\right)}^{2}\right.$ de riego (R) y drenaje (D), $\mathrm{y}$ de la diferencia entre el contenido de humedad volumétrica del sustrato $\left(\Delta \theta=\theta_{\mathrm{i}^{-}} \theta_{\mathrm{f}}\right)$ al inicio $\left(\theta_{\mathrm{i}}\right)$ $\mathrm{y}$ al final $\left(\theta_{\mathrm{f}}\right)$ de cada semana, según la ecuación $\mathrm{ET}_{\mathrm{c}}=\mathrm{R}-\mathrm{D} \pm \Delta \theta$.

El riego fue manejado independientemente para cada tratamiento con el objetivo de mantener el porcentaje de $\theta$ dentro del rango de AFD en el sustrato $\left(\theta_{\mathrm{CC}}-\theta_{5 \mathrm{kPa}}\right)$. El volumen de riego por planta/bolsa se estimó en función de i- un valor máximo de agotamiento preestablecido $(20 \%)$ del agua total disponible $(26,5 \%)$ en el sustrato (Figura 1), ii- del volumen de sustrato por planta/bolsa (Tabla 2) y iii- del porcentaje de drenaje preestablecido (15\%). Este último se estableció al considerar que la CE del agua de riego $\left(0,2 \mathrm{mS} \mathrm{cm}{ }^{-1}\right)$ utilizada permite usar hasta menos de $20 \%$ de drenaje.

Una vez determinado el volumen de agua a aplicar por planta en cada tratamiento, se calculó el tiempo de riego a partir del número de goteros por planta y del caudal promedio de los goteros. Este último fue obtenido mediante la determinación del coeficiente de uniformidad (>94\%) del sistema de riego (Christiansen 1942). El riego se controló con 2 programadores de riego, que mediante válvulas solenoides regó de forma independiente a cada tratamiento. $\mathrm{La}$ frecuencia de riego fue ajustada diariamente en función del volumen de drenaje y la $\theta$ para cada tratamiento, los cuales fueron medidos diariamente a las 6 a.m. antes del primer riego.

El porcentaje de drenaje se estimó como el cociente entre el volumen de agua drenada y el volumen de agua aplicada. Para esto, se colocaron 4 plantas en sus respectivas bolsas de cultivo dentro de una bandeja de madera $(1 \mathrm{~m} \times 0,3 \mathrm{~m}$ 
x $0,05 \mathrm{~m})$ hermetizada con plástico negro (230 $\mu \mathrm{m})$, con una leve pendiente (1\%) hacia uno de los extremos, donde se colocó un recipiente para recolectar el agua drenada. Para favorecer el drenaje se realizaron 4 agujeros de $4 \mathrm{~mm}$ de diámetro en la base y alrededor de la bolsa de cultivo. La bandeja se cubrió con plástico plata/negro para evitar la evaporación del agua drenada.

El contenido de humedad volumétrica en el sustrato $(\theta=\% \mathrm{v} / \mathrm{v})$ se midió con un sensor tipo "TDR" (Time Domain Reflectometry) conectado a un registrador de datos. La medición se realizó en 3 puntos distintos a media altura en cada una de las 4 bolsas por bandeja de drenaje, utilizando el valor promedio de $\theta$ de las 12 mediciones por repetición. A partir de la diferencia entre $\theta$ medida con el TDR y la $\theta_{\text {PMP }}$ obtenida en laboratorio (Figura 1), se obtuvo el valor real del \%ATD en el sustrato que, al multiplicarla por el volumen de sustrato, estimó el volumen (L) de ATD por planta/bolsa.

Eficiencia de uso del agua $\left(\mathrm{EUA}=\mathbf{k g}^{\mathrm{m}} \mathbf{m}^{-3}\right)$. La EUA se estimó para los rendimientos de fruto comercial (EUA $)$ y total (EUA $)$, como el cociente entre rendimiento $(\mathrm{kg})$ y la $\mathrm{ET}_{\mathrm{c}}\left(\mathrm{m}^{3}\right)$. El rendimiento comercial se determinó en 7 plantas de cada unidad de muestreo y se clasificó según el peso y las características del fruto en primera (I), segunda (II) y tercera (III), de acuerdo con la normativa nacional descrita por Quesada (2015). La categorías fueron: frutos I con peso mayor a $119 \mathrm{~g}$ sin deformaciones ni manchas, frutos II con peso entre 86-118 g con deformaciones y manchas leves, y frutos III con peso entre 51-85 g sin daños. Además, se cuantificó fruto de desecho, con peso menor 51 g y con daños físicos y mecánicos causados por insectos, hongos, necrosis apical, deshidratación y quema por sol, entre otros. El rendimiento total se obtuvo como la suma del rendimiento comercial más el desecho.

Análisis estadístico. Los datos experimentales fueron sometidos a un análisis de normalidad y homocedasticidad. Cuando se cumplieron dichos supuestos, los datos fueron sometidos a un análisis de varianza, para determinar si hubo efecto de la interacción o efectos independientes de ambos factores. Como no hubo efecto de la interacción $(\mathrm{p}>0,05)$ para ninguna de las variables de respuesta, se analizaron los efectos principales del factor o de ambos factores, que fueron estadísticamente significativos $(\mathrm{p}<0,05)$.

Para el análisis de la información se utilizó el siguiente modelo de análisis de varianza para bloques completos al azar según Di Rienzo et al. (2012):

Yijk $=\mu+\alpha \mathrm{i}+\beta \mathrm{j}+(\alpha \beta) \mathrm{ij}+\gamma \mathrm{k}+\varepsilon \mathrm{ijk}$, con $\mathrm{i}=$ $1,2, . . \mathrm{n} ; \mathrm{j}=1,2, \ldots \mathrm{n} ; \mathrm{k}=1,2, \ldots \mathrm{n}$ donde,

Yijk = variable de respuesta medida en la ijkésima unidad experimental.

$\mu=$ media general.

$\alpha \mathrm{i}=$ efecto del i-ésimo nivel del factor densidad de siembra.

$\beta \mathrm{j}=$ efecto del $\mathrm{j}$-ésimo nivel del factor volumen de sustrato.

$(\alpha \beta) \mathrm{ij}$ = efecto de la interacción entre el i-ésimo nivel del factor densidad de siembra y el j-ésimo nivel del factor volumen de sustrato.

$\gamma \mathrm{k}=$ efecto del k-ésimo bloque.

eijk $=$ error experimental asociado al ijk-ésimo valor medido en la ijk-ésima unidad experimental.

\section{RESULTADOS}

Para todas las variables de respuesta no se observaron tendencias diferentes entre ambas densidades de siembra conforme se incrementó el volumen de sustrato desde 7 hasta $21 \mathrm{~L}$, lo cual implica ausencia de interacción entre actores $(p>0,05)$. Ambos factores afectaron $(p<0,05)$, de forma independiente, con efecto de mayor magnitud sobre el drenaje y la $\mathrm{ET}_{\mathrm{c}}$ debido al factor densidad, y con magnitud similar sobre las EUAc y EUAt. El ATD y el rendimiento únicamente fueron afectados $(p<0,05)$, respectivamente, por el volumen de sustrato y la densidad de plantas (Tabla 4). 


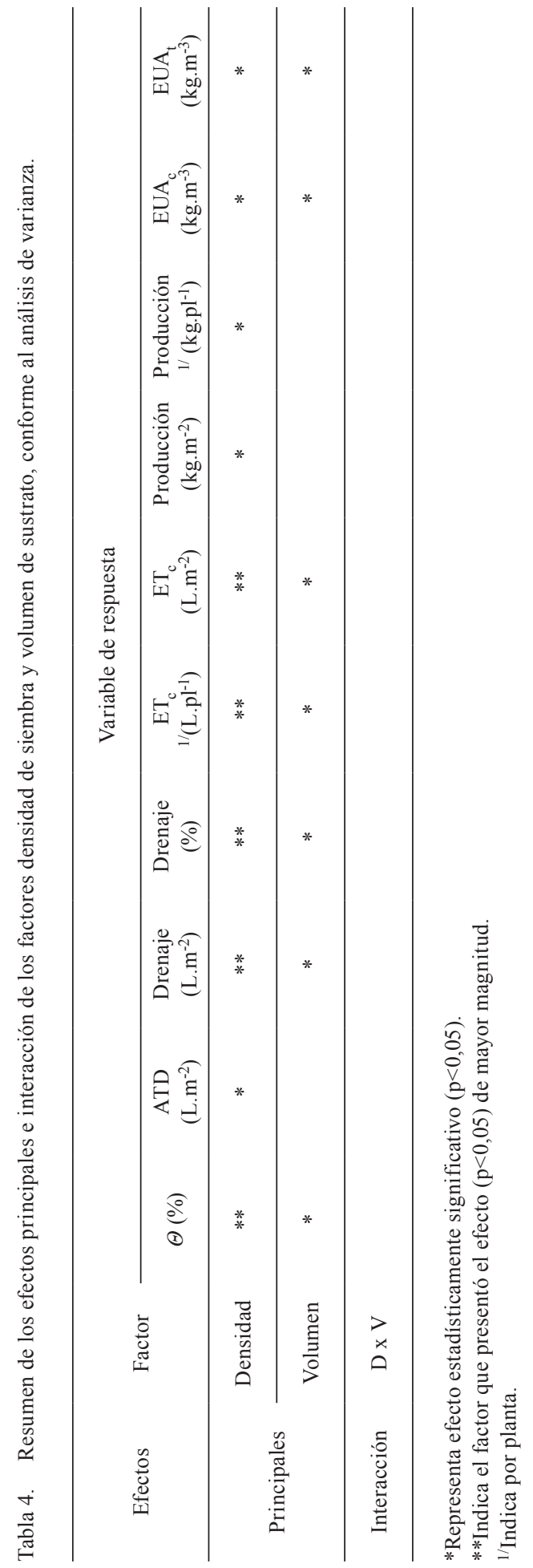


En el presente estudio, debido a la ausencia de interacción, en adelante únicamente se hace referencia al efecto independiente de la densidad de siembra y el volumen de sustrato. Inicialmente, se presenta información del riego, de la humedad volumétrica en el sustrato y del drenaje, ya que son componentes esenciales del balance hídrico para determinar la $\mathrm{ET}_{\mathrm{c}}$. Otra razón de consignar el riego como parte de los resultados, se basó en fundamentar el control del manejo del riego que permitió mantener la uniformidad en el contenido de humedad en el sustrato, y así, analizar el efecto de los tratamientos de volumen de sustrato y de la densidad de plantas por sí mismos.
Riego. El volumen de riego aportado por unidad de superficie $\left(\mathrm{L}^{\mathrm{m}} \mathrm{m}^{-2}\right)$ fue superior en los tratamientos de mayor densidad y con menor efecto, debido al volumen (Figura 3a, 3b). Las diferencias en los aportes de riego entre tratamientos (Figura 3a), debido a la densidad, empezaron a manifestarse a partir de la semana 5. El aporte inicial estuvo en valores cercanos a $0,5 \mathrm{~L} \cdot \mathrm{m}^{-2}$, incrementando progresivamente hasta alcanzar valores máximos de 7,4 L.m ${ }^{-2}$ en la semana 12 de cultivo (Figura 3a). En el riego acumulado (Figura 3b) no hubo tendencias diferentes entre ambas densidades de siembra conforme se incrementó el volumen de sustrato desde 7 hasta $21 \mathrm{~L}$.
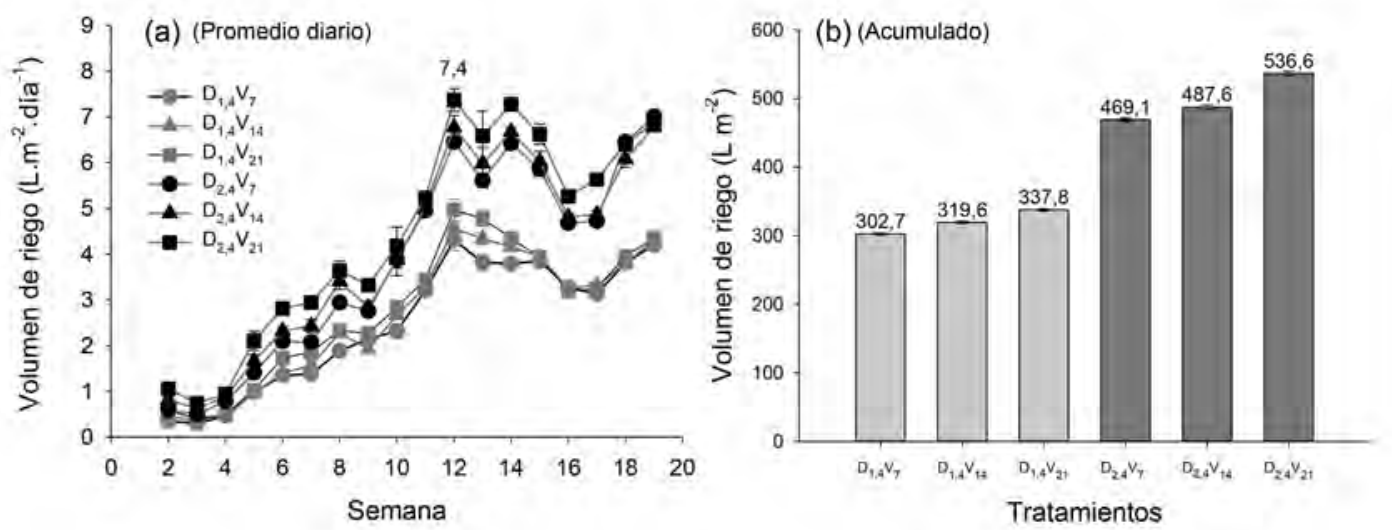

Figura 3 (a) Evolución semanal del aporte de riego promedio diario.

(b) Volúmenes de riego acumulado conforme al tratamiento.

El volumen de riego total incrementó desde 302 L.m ${ }^{-2}$ hasta 536 L.m ${ }^{-2}$ al pasar del tratamiento $\mathrm{D}_{1,4} \mathrm{~V}_{7}$ a $\mathrm{D}_{2,4} \mathrm{~V}_{21}$, respectivamente. El incremento promedio al aumentar la densidad de 1,4 plantas $\mathrm{m}^{-2}$ $\left(320 \mathrm{~L} \cdot \mathrm{m}^{-2}\right)$ a 2,4 plantas $\mathrm{m}^{-2}\left(536,6 \mathrm{~L} \cdot \mathrm{m}^{-2}\right)$ fue de $56 \%$; mientras que al pasar de $7 \mathrm{~L}$ de sustrato por planta $\left(385,9\right.$ L.m $\left.{ }^{-2}\right)$ a $21 \mathrm{~L}\left(437,2 \mathrm{~L} . \mathrm{m}^{-2}\right)$ el incremento promedio fue solamente un $13 \%$.

Humedad volumétrica $(\theta)$ en el sustrato. Durante el ciclo de cultivo, la $\theta$ en el sustrato se mantuvo relativamente constante, sin mayores diferencias entre tratamientos (Figura 4a) y dentro del rango de AFD entre $\theta_{\mathrm{CC}}(57,4 \%)$ y $\theta_{5 \mathrm{kPa}}(35,2 \%)$ (Figura 1$)$. Consecuentemente, no hubo efecto de la interacción $(\mathrm{p}>0,05)$, ni efectos independientes de la densidad de siembra y el volumen de sustrato sobre el porcentaje de $\theta$. Los promedios de $\theta$ en los diferentes tratamientos, entre $45 \%$ a $49 \%$, oscilaron dentro del rango de AFD de la fibra de coco (Figura 4b). 


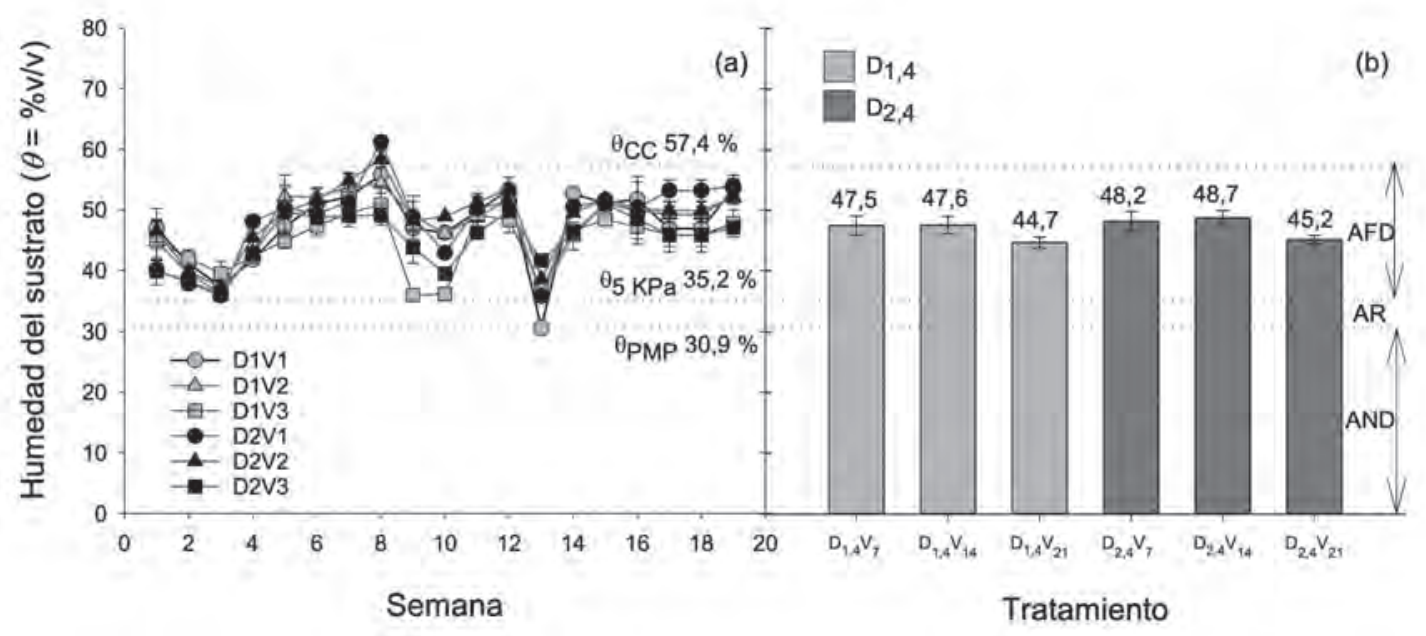

Figura 4 (a) Evolución semanal del contenido promedio de humedad volumétrica (\%) en el sustrato. (b) Promedio global según tratamiento.

Agua total disponible (ATD) en el sustrato. El volumen de ATD por planta y por $\mathrm{m}^{2}$ no fue afectado por la interacción entre ambos factores. A nivel de planta, el ATD únicamente fue afectada $(p<0,05)$ por el volumen de sustrato (Figura 5a). Independientemente de la densidad, el ATD fluctuó desde 1,2 L de ATD en los tratamientos con $7 \mathrm{~L}$ de sustrato hasta 3,0 L de ATD. planta $^{-1}$ al utilizar $21 \mathrm{~L}$ de sustrato.planta ${ }^{-1}$, lo cual representó un apreciable incrementó de 149\%. Sin embargo, al analizar el ATD por área (Figura $5 b$ ), a diferencia del ATD.planta ${ }^{-1}$, se observó que dicha variable fue afectada significativamente por ambos factores $(p<0,05)$ con una magnitud similar. El aumento la densidad de 1,4 a 2,4 plantas. $\mathrm{m}^{-2}$ representó un incremento de un $80 \%$; mientras que al incrementar el volumen de sustrato de $7 \mathrm{~L}$ a $21 \mathrm{~L}$, el ATD incrementó un 83\%.
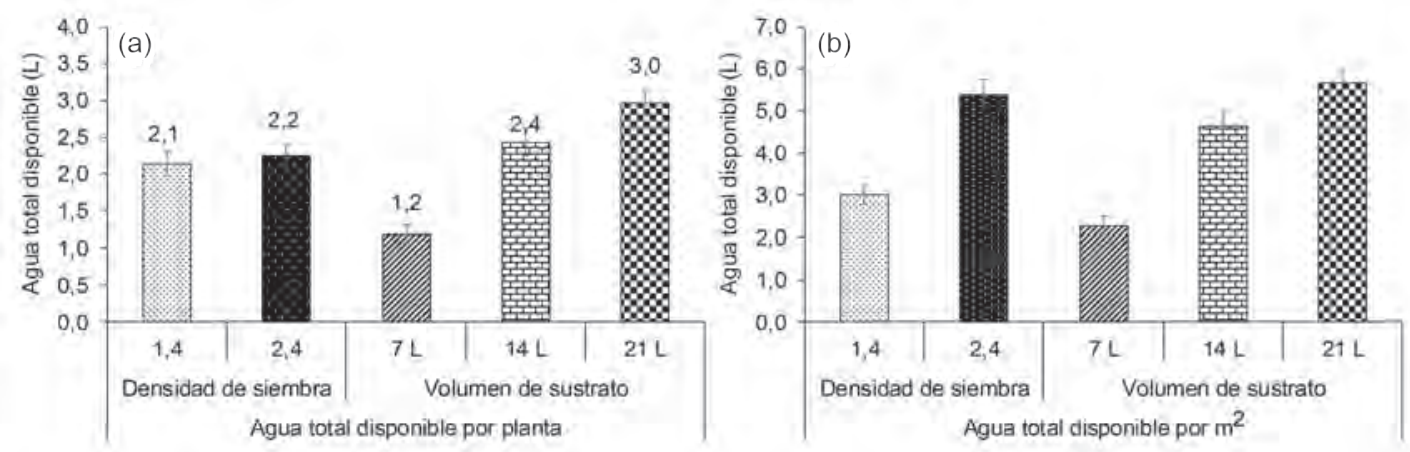

Figura 5 Agua total disponible por planta.

(a) Coforme tratamiento combinado.

(b) Conforme volumen de sustrato por planta. 
Volumen y porcentaje de drenaje. La densidad de siembra y el volumen de sustrato afectaron $(\mathrm{p}<0,05)$ de forma independiente, sin interacción, a ambas variables, con el mayor efecto debido al factor densidad. En el caso del volumen de drenaje, este incrementó un $89 \%$ al pasar de una condición de baja a alta densidad; mientras que al cambiar el volumen de $7 \mathrm{~L}$ a $21 \mathrm{~L}_{\text {de }}$ sustrato.planta ${ }^{-1}$, el volumen de drenaje incrementó tan solo un 17\% (Figura 6a).
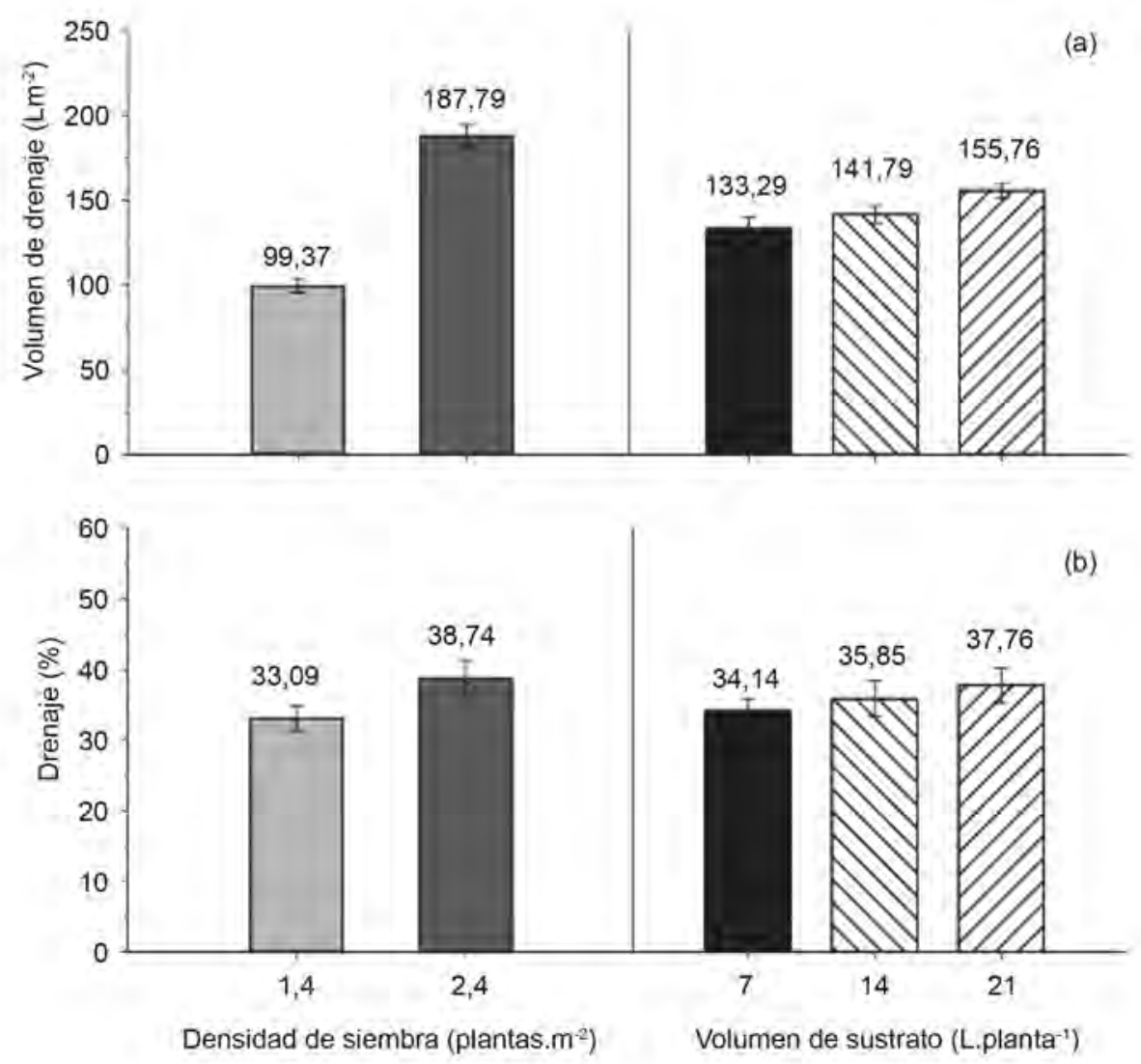

Figura 6 (a) Efectos principales de la densidad de siembra y volumen de sustrato sobre el volumen total drenado. (b) Porcentajes de drenaje.

El porcentaje de drenaje (Figura $6 b$ ) tuvo comportamiento similar al observado en el caso del volumen de drenaje (Figura 6a), ya que mostró una tendencia al incremento conforme aumentó la densidad de plantación y el volumen de sustrato. La densidad tuvo un efecto ligeramente superior al volumen de sustrato, ya que el porcentaje de drenaje incrementó un $17 \%$, al aumentar la densidad de 1,4 a 2,4 plantas. $\mathrm{m}^{-2}$, y un $11 \%$ al incrementar el volumen de sustrato de $7 \mathrm{~L}$ a $21 \mathrm{~L}$ (Figura 6b).

Evapotranspiración del cultivo (ET $)$. $\mathrm{La} \mathrm{ET}_{\mathrm{c}}$ por $\mathrm{m}^{-2}$ y por planta ${ }^{-1}$ no mostraron tendencias diferentes entre ambas densidades de siembra conforme incrementó el volumen 
de sustrato.planta ${ }^{-1}(\mathrm{p}>0,05)$. Sin embargo, dichas variables fueron afectadas por ambos factores $(p<0,05)$, con mayor magnitud debido al factor densidad.

Los efectos principales de la densidad de siembra y el volumen de sustrato, por área y por planta, se presentan en la Figura 7. Al analizar dichos efectos sobre la $\mathrm{ET}_{\mathrm{c}} \cdot$ planta $^{-1}$, indistintamente del volumen del contenedor, se redujo un $18 \%$ al aumentar la densidad de 1,4 plantas. $\mathrm{m}^{-2}$ a 2,4 plantas. $^{-2}$ (Figura 7a). Por otra parte, contrario al consumo por planta, la $\mathrm{ET}_{\mathrm{c}}$ por área, aumentó un $41 \%$ al incrementar la densidad de siembra de 1,4 a 2,4 plantas. $\mathrm{m}^{-2}$.
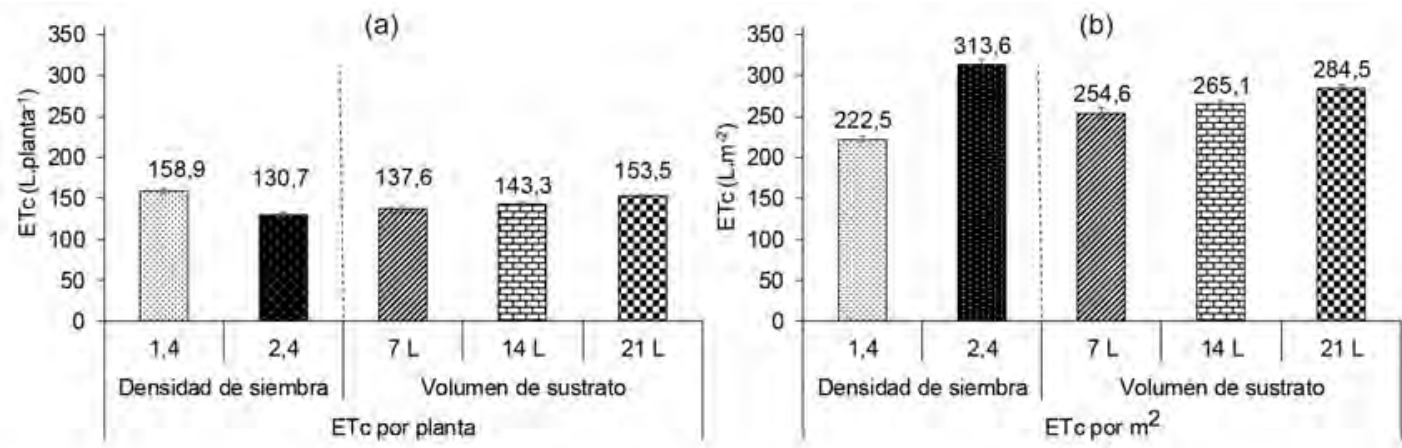

Figura 7 (a) Efectos principales de la densidad de siembra y el volumen de sustrato sobre la evapotranspiración total del cultivo $\left(\mathrm{ET}_{\mathrm{c}}\right.$ ), por planta.

(b) Efectos por área.

Respecto a los efectos del volumen de sustrato, tanto la $\mathrm{ET}_{\mathrm{c}}$ por planta como por área, incrementaron en magnitud similar, en un promedio de $12 \%$ conforme aumentó el volumen desde $7 \mathrm{~L}$ a $21 \mathrm{~L}$ de sustrato/planta (7b).

Considerando la importancia de la disponibilidad de datos de referencia acerca del consumo de agua $\left(\mathrm{ET}_{\mathrm{c}}: \mathrm{L}_{\mathrm{m}} \mathrm{m}^{-2}\right)$ para la producción, en la Figura 8 se presentan valores promedio diarios de $\mathrm{ET}_{\mathrm{c}}$ por tratamiento combinado, donde se observa una tendencia a incrementar la $\mathrm{ET}_{\mathrm{c}}$ conforme aumentó la densidad de siembra y el volumen de sustrato. Durante el ciclo del cultivo, en todos los tratamientos la $\mathrm{ET}_{\mathrm{c}}$ diaria $\left(\mathrm{L} . \mathrm{m}^{-2}\right)$ incrementó progresivamente desde la semana $1\left(0,2 \mathrm{~L} \cdot \mathrm{m}^{-2} \cdot \mathrm{dí}^{-1}\right)$ hasta alcanzar valores máximos en la semana 14 , con 4,9 L.m $\mathrm{m}^{-2}$.día $\mathrm{a}^{-1}$ en los tratamientos de mayor densidad $\left(\mathrm{D}_{2,4} \mathrm{~V}_{21}\right)$ y de 3,0 L.m $\mathrm{m}^{-2} \cdot$ día $^{-1}$ en la menor densidad $\left(\mathrm{D}_{1,4} \mathrm{~V}_{7}\right)$, con una tendencia a disminuir en el resto del ciclo (Figura 8a). 


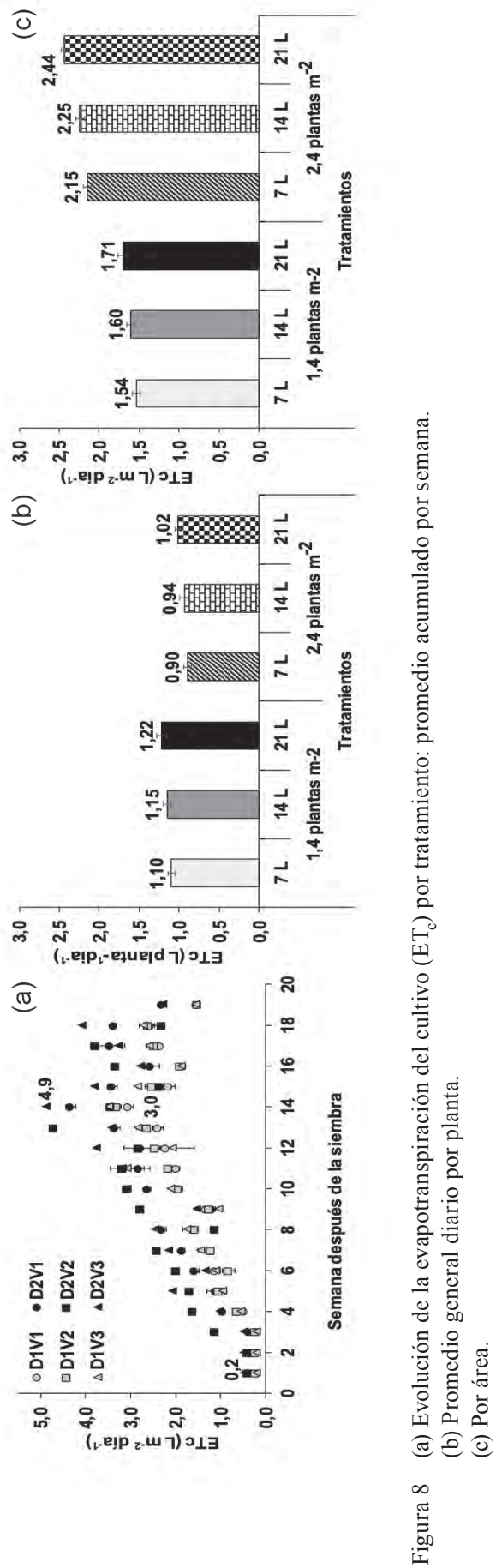

Agronomía Costarricense 44(1): 43-64. ISSN:0377-9424 / 2020 
En relación con los promedios globales de todo el ciclo (Figura 8b, 8c), la $\mathrm{ET}_{\mathrm{c}}$.planta ${ }^{-1}$ disminuyó en los tratamientos de mayor densidad (Figura 8b). Consecuentemente, las ET máxima y mínima se obtuvieron, respectivamente, en los tratamientos con 1,4 plantas y $21 \mathrm{~L}$ de sustrato/planta (1,22 L.planta ${ }^{-1} \cdot$ día $\left.^{-1}=\mathrm{D}_{1,4} \mathrm{~V}_{21}\right)$, y con 2,4 plantas. $\mathrm{m}^{-2}$ y $7 \mathrm{~L}$ de sustrato/planta $(0,9$ L.planta ${ }^{-1}$.día ${ }^{-1}=\mathrm{D}_{2,4} \mathrm{~V}_{7}$ ).

Por otra parte, el consumo por área (Figura 8c) incrementó conforme aumentó la densidad de siembra y el volumen sustrato, fluctuando desde un mínimo de 1,54 L.m ${ }^{-2}$. día $^{-1}$ en los tratamientos de menor volumen $\left(\mathrm{D}_{1,4} \mathrm{~V}_{7}\right)$ hasta un máximo de $2,44 \mathrm{~L} \cdot \mathrm{m}^{-2} \cdot$ día $^{-1}$ en los tratamientos con $21 \mathrm{~L}^{\text {de sustrato.planta }}{ }^{-1}$ $\left(\mathrm{D}_{2,4} \mathrm{~V}_{21}\right)$.

Rendimiento. Los componentes de rendimiento en número y peso de frutos comercial y total, por planta y por área, únicamente fueron afectados $(\mathrm{p}<0,05)$ por la densidad de siembra, sin interacción entre ambos factores. En la Figura 9 se presentan los efectos principales de la densidad de plantación sobre dichos componentes del rendimiento. A nivel de planta, el peso de frutos comercial y total fue mayor en el cultivo de menor densidad, debido a que al disminuir la densidad, incrementó el número de frutos.planta ${ }^{-1}$ en comparación a la densidad de 2,4 plantas. m $^{-2}$ (Figura 9a). Sin embargo, al analizar el rendimiento por área, el incremento en el número de plantas al aumentar la densidad de plantación a 2,4 plantas. $\mathrm{m}^{-2}$, incrementó la cantidad de frutos. $\mathrm{m}^{-2}$ y consecuentemente el peso de frutos. $\mathrm{m}^{-2}$ (Figura 9b). Independientemente del volumen de sustrato, al aumentar la densidad desde 1,4 a 2,4 plantas. $\mathrm{m}^{-2}$, incrementó la producción comercial un promedio de $30 \%$, equivalente a $2,7 \mathrm{~kg} \cdot \mathrm{m}^{-2}$ y 22 frutos. $\mathrm{m}^{-2}$.
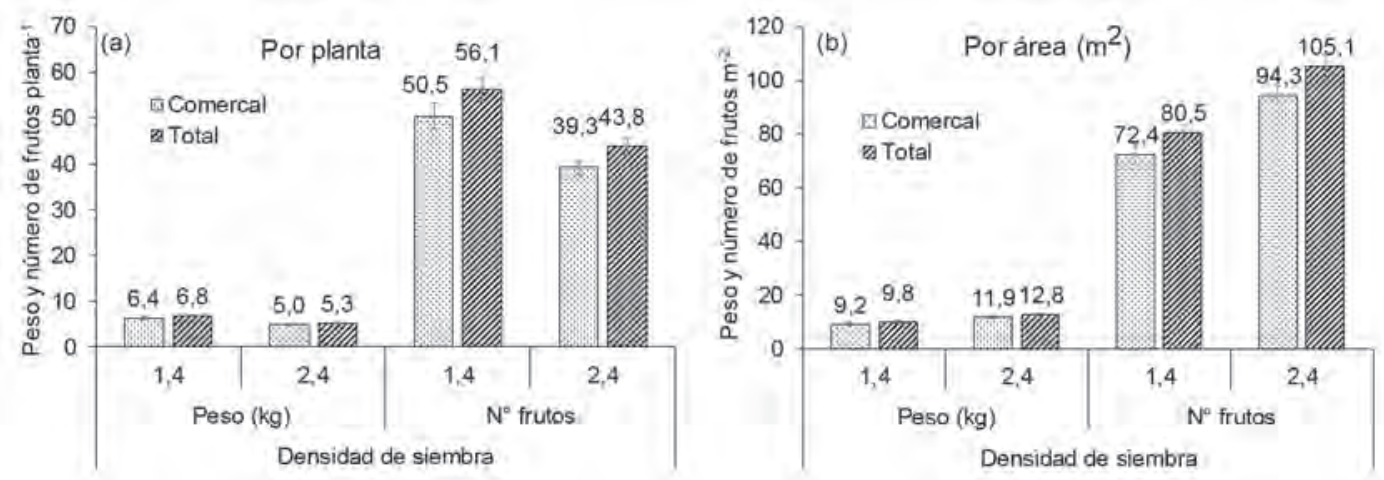

Figura 9 (a) Efectos principales de la densidad de siembra, según los componentes de rendimiento de número y peso de frutos comercial y total por planta.

(b) Efectos por unidad de superficie.

Eficiencia de uso de agua. La eficiencia de uso de agua en producción de fruto comercial $\left(\right.$ EUA $\left._{c}\right)$ y total $\left(\right.$ EUA $\left._{t}\right)$ no fue afectada $(\mathrm{p}<0,05)$ por la interacción entre densidad de siembra y el volumen de sustrato; mientras que hubo efecto $(p<0,05)$ independiente, con similar magnitud, de ambos factores, cuyos efectos principales se presentan en la Figura 10. En general, dichas eficiencias incrementaron al disminuir la densidad de siembra y el volumen de sustrato. Indistintamente del volumen de sustrato, al pasar de una densidad de 1,4 a 2,4 plantas. $\mathrm{m}^{-2}$, ambas eficiencias disminuyeron un promedio de $11 \%$. En el volumen de $21 \mathrm{~L}$ de sustrato, ambas eficiencias 
fueron significativamente menores $(\mathrm{p}<0,05)$ que en los tratamientos de 7 y $14 \mathrm{~L}$ de sustrato.plan$\mathrm{ta}^{-1}$, los cuales fueron estadísticamente iguales $(\mathrm{p}>0,05) . \mathrm{Al}$ incrementar el volumen de sustrato de $7 \mathrm{~L}$ a $21 \mathrm{~L}_{\text {sustrato.planta }}{ }^{-1}$, ambas eficiencias disminuyeron un promedio de $13,5 \%$.

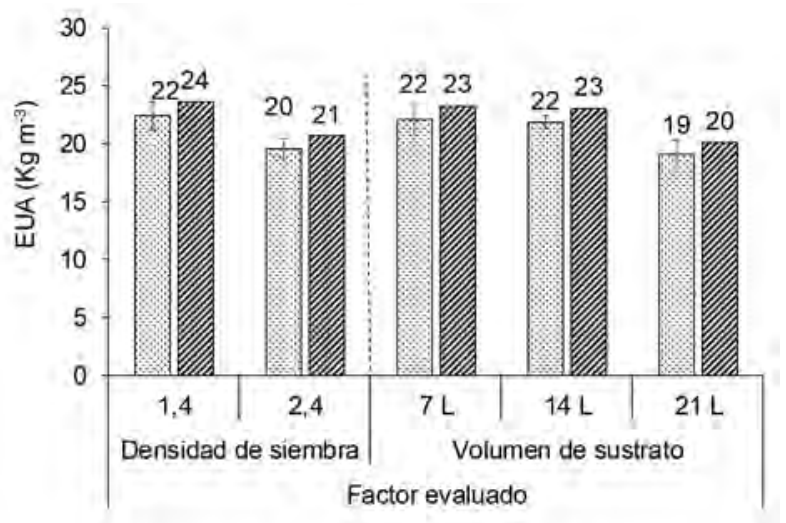

Figura 10 Efectos principales de la densidad de siembra y del volumen de sustrato sobre la eficiencia de uso de agua en rendimiento comercial $\left(\mathrm{EUA}_{\mathrm{c}}\right)$ y total $\left(\mathrm{EUA}_{\mathrm{t}}\right)$.

\section{DISCUSIÓN}

Debido a la ausencia de interacción entre factores, a continuación se analizan los efectos principales de la densidad de siembra y volumen de sustrato que fueron estadísticamente significativos sobre las variables de respuesta.

$\mathrm{La} \mathrm{ET}_{\mathrm{c}}$ fue afectada en mayor magnitud por la densidad de siembra que por el volumen de sustrato. En cultivo en sustrato, el principal componente de la $\mathrm{ET}_{\mathrm{c}}$ es la transpiración, ya que la evaporación se reduce a valores cercanos a cero (Allen et al. 2006). En este ensayo, la superficie húmeda y expuesta del sustrato se redujo sustancialmente al estar cubierto con plástico, donde solo una pequeña área por donde emerge la planta estuvo expuesta a evaporación. El mayor efecto de la densidad de plantas, probablemente, se debió a que dicho factor afecta parámetros de crecimiento de las plantas, tales como el área foliar (AF) y en el tamaño y numero de hojas, que están directamente asociados a la transpiración del cultivo (Taiz y Zeiger 2006). Diferentes personas autoras han demostrado que al incrementar la densidad de siembra, el AF y el número de hojas por planta, disminuye (Aminifard et al. 2010) pero incrementan por unidad de superficie. Otras personas autoras han demostrado que el incremento en la densidad de plantación tiene como consecuencia un aumento en la transpiración, debido a un mayor IAF (Qiu et al. 2013, Di Benedetto y Tognetti 2016). En chile dulce bajo túneles de plástico, Jolliffe y Gaye (1995) encontraron que el IAF incrementó desde $1,5 \mathrm{~m}^{2} \cdot \mathrm{m}^{-2}$ en 1,4 plantas. $\mathrm{m}^{-2}$ hasta $4 \mathrm{~m}^{2} \cdot \mathrm{m}^{-2}$ en 11,1 plantas. $\mathrm{m}^{-2}$; mientras que Aminifard et al. (2010) determinaron un incremento en el número de hojas a mayor densidad de plantas. $\mathrm{m}^{-2}$.

Coincidente con lo anterior, en el presente estudio, a mayor densidad la $\mathrm{ET}_{\mathrm{c}} \cdot$ planta $^{-1}$ disminuyó, pero incrementó por área $\left(\mathrm{L} \cdot \mathrm{m}^{-2}\right)$. Probablemente, al incrementar la densidad de siembra mediante la reducción de la distancia entre plantas de 0,55 a 0,3 m, incrementó el AF y el número de hojas por $\mathrm{m}^{-2}$, contribuyendo así, a una mayor transpiración.

La literatura acerca de la $\mathrm{ET}_{\mathrm{c}}$ y la EUA relacionada con la densidad de siembra y el volumen de sustrato en chile dulce bajo invernadero, es escaza; y específicamente en Costa Rica hay 
un apreciable vacío de información. En cultivos como el tomate en invernadero en suelo, Qiu et al. (2013) encontraron que la $\mathrm{ET}_{\mathrm{c}}$ incrementó un $11 \%$ al aumentar la densidad de 3,1 a 5,6 plantas.m ${ }^{-2}$. Por otra parte, Silva et al. (2010) determinaron que el efecto de la densidad de plantación sobre el crecimiento del cultivo de chile dulce está principalmente determinado por la distancia entre plantas y no por la distancia entre hileras, ya que se anula el efecto de competencia entre hileras. En dicha literatura se encontró que el AF y los contenidos de MS por unidad de área incrementaron conforme aumentó la densidad de siembra al reducir la distancia entre plantas desde $0,4 \mathrm{~m}$ hasta $0,2 \mathrm{~m}$, sin efectos debido a la distancia entre hileras.

El volumen de sustrato afectó los volúmenes de $\mathrm{ET}_{\mathrm{c}}$, de drenaje y de ATD, y la EUA, pero no tuvo efecto sobre el rendimiento. $\mathrm{La} \mathrm{ET}_{\mathrm{c}} \cdot \mathrm{m}^{-2}$ y ET . $_{\text {planta }}{ }^{-1}$ disminuyeron conforme se redujo el volumen de sustrato por planta, probablemente debido a una reducción de la traspiración, relacionado con una reducción del AF del cultivo y del contenido de ATD en el sustrato. En este estudio, con el objetivo de mantener la $\theta$ en el rango de AFD en el sustrato (Figura 2), fue necesario incrementar el aporte de agua conforme aumentó el volumen de sustrato por planta, lo cual a la vez estuvo relacionado con el incremento de los volúmenes de drenaje y de ATD. Al respecto, Bar-Tal et al. (1995) demostraron que en chile hidropónico bajo invernadero al reducir el volumen del contenedor se restringe el crecimiento radical y el AF y consecuentemente la transpiración.

Según Moreno-Reséndez et al. (2015), una reducción del volumen de sustrato limita el crecimiento de la raíz, debido a la reducción en la disponibilidad de oxígeno, agua y nutrientes, que consecuentemente afecta parámetros de crecimiento tales como el AF y el tamaño y número de hojas. En la presente investigación, un menor volumen de sustrato por planta, probablemente, afectó la concentración oxígeno en la rizosfera. Al respecto, Vence et al. (2013) señala que cuando se limita el volumen de sustrato, el consumo de oxigeno es mayor, mientras que la capacidad de aireación se reduce, debido al incremento en la densidad radical por unidad de volumen. Por otra parte, la temperatura ambiental afecta la temperatura del medio de cultivo en mayor magnitud, conforme se reduce el volumen de sustrato, que a su vez afecta la concentración de oxígeno en la rizosfera (Hurley et al. 1998).

No obstante, los potenciales efectos negativos de un menor volumen de sustrato, no afectaron el rendimiento por planta, sino por área, debido al incremento en el número de plantas $\mathrm{m}^{-2}$. Probablemente, el híbrido dulcitico, por ser mejorado localmente para las condiciones específicas del trópico, podría presentar una posible plasticidad genética del sistema radical que le permite una mejor adaptación a volúmenes reducidos de sustrato.

Independiente de la densidad de plantas, el ATD por planta incrementó al aumentar el volumen de sustrato, lo cual estuvo relacionado con una mayor $\mathrm{ET}_{\mathrm{c}}$, debido a incrementos en la transpiración. Al respecto, diferentes estudios han demostrado que el aumento del volumen de sustrato favorece el crecimiento del cultivo (Poorter et al. 2012, Luo et al. 2015, Graham y Wheeler 2015) y consecuentemente la $\mathrm{ET}_{\mathrm{c}}$. Otras personas autoras han demostrado que a menor volumen de sustrato se reduce la transpiración, debido a una reducción del AF, el número y tamaño de la hoja, peso seco de raíz y en la respuesta estomática (NeSmith et al. 1992, Kharkina et al. 1999, Ismail et al. 2002).

En los cultivos sin suelo, el volumen de sustrato no está distribuido por unidad de superficie $\left(\mathrm{L} . \mathrm{m}^{-2}\right)$ y el sistema radicular de cada planta explora un volumen de sustrato que está determinado por las dimensiones del contenedor $\left(\right.$ L.planta ${ }^{-1} \cdot$ bolsa $\left.^{-1}\right)$. Diversos estudios han demostrado que al incrementar el volumen de sustrato, aumenta el volumen de ATD para la planta (Hurley et al. 1998). Al respecto, Moral et al. (2014) demostraron que un mayor contenido de ATD.planta ${ }^{-1}$ es un parámetro clave en la morfología radicular, ya que genera mayor longitud 
y área radicular, pero menor diámetro de raíces, lo que indica una formación más eficiente de pelos radiculares. Esto provee a la planta mayor capacidad de absorción de agua y de nutrientes y, por tanto, mayor crecimiento foliar. En cultivo de chile dulce bajo invernadero, Fernández et al. (2005) demostraron que al aumentar el volumen de ATD para la planta mediante tratamientos con dosis crecientes de riego, consecuentemente aumentó la $\mathrm{ET}_{\mathrm{c}} \mathrm{y}$ disminuyó la EUA.

Durante el ciclo del experimento, el manejo independiente y diferenciado del riego, de acuerdo con el volumen de sustrato por planta, permitió mantener el porcentaje de $\theta$ dentro del rango de AFD en el sustrato. Para mantener la $\theta$ en el rango de AFD, fue necesario incrementar el aporte de agua conforme se incrementó el volumen de sustrato por planta (Figura 3b). Dicho comportamiento reflejó la interacción sustratoplanta-atmósfera, ya que a mayor volumen de riego, como consecuencia de mayor volumen de sustrato.planta ${ }^{-1}$, incrementó el volumen de ATD.planta ${ }^{-1}$, y consecuentemente, hubo mayores volúmenes de drenaje y de $\mathrm{ET}_{\mathrm{c}}$.

Un mayor volumen de drenaje, en los tratamientos de más volumen de sustrato, coinciden con estudios realizados por Abdel-Maugoud et al. (2006), quienes aplicaron diferentes volúmenes de riego en chile dulce cultivado en lana de roca, obteniendo un $8 \%$ menos de drenaje en el tratamiento con menor ATD.planta ${ }^{-1}$.

\section{Rendimiento y eficiencia de uso de} agua. El peso medio de fruto no fue afectado por ninguno de los factores estudiados, coincidente con estudios realizados por Cavero et al. (2001) y Cruz et al. (2009) en chile dulce. Por otra parte, los componentes de rendimiento número y peso de frutos, únicamente fueron afectados por la densidad de siembra. A mayor densidad, el número y rendimiento de frutos comercial y total disminuyó por planta; sin embargo, incrementó por unidad de superficie, lo cual coincide con diversos estudios realizados en chile dulce (Hossein 2012, Cavero et al. 2001, De Freitas et al. 2017, Ngozi y Chidera 2017). Rangel (2016) demostró que el rendimiento en chile dulce incrementó de 2,25 a 4,26 y kg. $\mathrm{m}^{-2}$ al aumentar la densidad de 2,8 a 5,3 plantas. $\mathrm{m}^{-2}$, respectivamente; mientras que Hossein (2012) reportó que los rendimientos aumentaron de $4,9 \mathrm{~kg} \cdot \mathrm{m}^{-2}$ en 3,3 plantas. $\mathrm{m}^{-2}$ hasta $12,9 \mathrm{~kg} \cdot \mathrm{m}^{-2}$ en 10 plantas. $\mathrm{m}^{-2}$. En cultivo de chile dulce bajo invernadero se han reportado rendimientos desde 32,5 ton.ha ${ }^{-1}$ (NafarrateRamos et al. 2016) hasta 81,5 t.ha-1 (Dafgan y Abak 2003). En este experimento, se obtuvo un rendimiento comercial superior, con un máximo de 120 t.ha ${ }^{-1}$ a 2,4 plantas.m ${ }^{-2}$ y hasta 56 frutos. planta $^{-1}$ a una densidad de 1,4 plantas. $\mathrm{m}^{-2}$.

Entre las alternativas para mejorar la EUA, se incluyen todas aquellas prácticas de manejo del cultivo orientadas a reducir el consumo de agua y a aumentar el rendimiento del cultivo (Ritchie y Basso 2008). Debido al sistema hidropónico utilizado en este estudio, la evaporación fue insignificante, por lo que el consumo de agua correspondió básicamente a transpiración. Ritchie y Basso (2008) en una revisión exhaustiva, demostraron que la densidad de plantas y el aporte de agua influyen en el rendimiento, el cual mantiene una relación lineal con la EUA. Considerando que la EUA es la relación entre producción $\left(\mathrm{kg} \cdot \mathrm{m}^{-2}\right)$ y $\mathrm{ET}_{\mathrm{c}}\left(\mathrm{L} \cdot \mathrm{m}^{-2}\right)$, se espera que un mayor rendimiento obtenido en la densidad de 2,4 plantas. $\mathrm{m}^{-2}$, resultara en una mayor EUA. Sin embargo, una menor densidad a 1,4 planta. $\mathrm{m}^{-2}$ contribuyó a reducir la $\mathrm{ET}_{\mathrm{c} .} \mathrm{m}^{-2}$ en un promedio de $41 \%$, lo cual ayudó a incrementar un $11 \%$ la EUA, en comparación a la densidad de 2,4 planta. $\mathrm{m}^{-2}$. En el cultivo de mayor densidad de plantas, aunque el rendimiento por área aumentó debido al incremento en el número de plantas, la magnitud del incremento, debido al efecto del factor densidad, fue mayor para la $\mathrm{ET}_{\mathrm{c}}$ que para el rendimiento.

Los valores de EUA obtenidos a menor densidad, en el presente estudio (21,2 a 25,3 $\mathrm{kg} . \mathrm{m}^{-3}$ ), fueron superiores a los reportados por Fernández et al. (2007) en el cultivo de chile dulce bajo invernadero en Almería, España (13,1 kg. $\mathrm{m}^{-3}$ ) y por Arévalo (2015) en Comayagua, Honduras. Aunque el factor volumen no influyó directamente en los rendimientos, el volumen 
promedio de sustrato por unidad de superficie fue mayor en la densidad de 2,4 planta. $\mathrm{m}^{-2}$ $\left(33,6 \mathrm{~L} \cdot \mathrm{m}^{-2}\right)$ que en 1,4 planta.m ${ }^{-2}\left(19,6 \mathrm{~L} \cdot \mathrm{m}^{-2}\right)$. Por tanto, para mantener la $\theta$ dentro del rango de AFD en el sustrato, en los tratamientos de 2,4 planta. $\mathrm{m}^{-2}$, fue necesario aplicar mayores volúmenes de riego, lo cual, consecuentemente, incrementó los volúmenes de ATD, el drenaje y la $\mathrm{ET}_{\mathrm{c}}$, reduciendo la EUA.

Xu and Kafkafi (2001), al evaluar el efecto del volumen de sustrato sobre el rendimiento, encontraron que al incrementar de 9 a $33 \mathrm{~L} . p l a n t a^{-1}$, el rendimiento aumentó; mientras que Karam et al. (2009), en chile "Bell pepper", demostraron que al aumentar el volumen de ATD en el sustrato, incrementó el rendimiento, debido a una mayor eficiencia de uso de la radiación. Sin embargo, en este estudio al incrementar el volumen de sustrato, el potencial efecto positivo sobre la producción no se manifestó. Por tanto, un hallazgo relevante es el comportamiento característico del genotipo del híbrido Dulcitico en respuesta al volumen del contenedor y a la densidad de plantas. Por primera vez, se reporta la $\mathrm{ET}_{\mathrm{c}}$ obtenida por un método directo (lisímetro), el rendimiento de frutos y la eficiencia física de uso del agua, bajo un manejo hidropónico y en invernadero.

En resumen, un menor volumen de sustrato no incrementó los rendimientos por área, sin embargo, mejoró la EUA al reducir los volúmenes de riego, de $\mathrm{ET}_{\mathrm{c}} \mathrm{y}$ de drenaje; mientras que una mayor densidad de siembra mejoró los rendimientos $\left(\mathrm{k} \cdot \mathrm{m}^{-2}\right)$, pero redujo la EUA, debido a un mayor gasto de agua como consecuencia de mayores volúmenes de riego, de $\mathrm{ATD}$, de $\mathrm{ET}_{\mathrm{c}} \mathrm{y}$ de drenaje. Aun cuando la mayor densidad de siembra elevó los rendimientos en un 30\% (2 $\mathrm{kg} . \mathrm{m}^{-2}$ ), es importante determinar la densidad óptima económica, en la cual se da la mayor relación beneficio/costo. En este sentido, Camacho (2017), mediante un análisis de presupuestos parciales, determinó que el tratamiento de 1,4 plantas. $\mathrm{m}^{-2}$ y $7 \mathrm{~L}$ de sustrato presentó el mayor beneficio neto, debido a una mayor magnitud en la reducción en los costos. Coincidentemente, dicho tratamiento presentó la mayor EUA.

\section{LITERATURA CITADA}

Abdel-Maugoud, AMR; Sassine, YN; Ghora, Y; Heuvelink, E. 2006. Independent effect of water content in rockwool ${ }^{\circledR}$ on water use, growth and production of greenhouse sweet pepper European Journal of Scientific Research 15:235-234.

Allen, RG; Pereira, LS; Raes, D; Smith, M. 2006. Evapotranspiración del cultivo. Guías para la determinación de los requerimientos de agua de los cultivos. Estudio FAO-56. Riego y Drenaje. Roma, Italia. $323 \mathrm{p}$.

Aminifard, MH; Aroiee, H; Karimpour, S; Nemati, H. 2010. Growth and yield characteristics of paprika pepper (Capsicum annum L.) in response to plant density. Asian Journal of Plant Sciences 9(5):276-280.

Arèvalo, G. 2015. Determinación de la eficiencia de manejo de nutrientes y agua en tres variedades de pimiento de color (Capsicum annuum L.) bajo hidroponía en condiciones de invernadero en Mulacagua, Comayagua, Honduras. Tèsis doctoral. Almería, España, Universidad de Almería. 193 p.

Bar-Tal, A; Feigin, A; Sheinfeld, S; Rosenberg, R; Sternbaum, B; Rylski, I; Pressman, E. 1995. Root restriction and $\mathrm{N}-\mathrm{NO}$, solution concentration effects on nutrient uptake, transpiration and dry matter production of tomato. Scientia Horticulturae 63:195-208.

Cabrera, J. 2009. Evaluación del consumo de agua y nutrientes en chile (Capsicum annuиm), variedades Taranto, Zidenka y Sympathy en hidroponía y condiciones de altura en Mulacagua, Comayagua, Honduras. Tesis Lic. Honduras, Zamorano. 31 p.

Camacho, G. 2017. Evaluación económica para la producción del híbrido de chile dulce (Capsicum annuum) "Dulcitico" en invernadero, mediante el enfoque de presupuestos parciales, en la Estación Experimental Agrícola Fabio Baudrit Moreno, Alajuela, Costa Rica. Tesis de Lic. San José, Costa Rica, Universidad de Costa Rica. 148 p.

Cavero, J; Gil Ortega, R; Gutierrez, M. 2001. Plant density affects yield, yield components, and color of directseeded paprika pepper. Hort. Science 36(1):76-79.

Christiansen, JE. 1942. Irrigation by Sprinkling. California Agricultural Experiment Station. Bulletin $\mathrm{N}^{\mathrm{o}} 670.126 \mathrm{p}$.

Cruz, N; Sánchez, F; Ortiz, CJ; Mendoza, CM del C. 2009. Altas densidades con despunte temprano en rendimiento y período de cosecha en chile pimiento. Agricultura Técnica en México 35(1):73-80.

Dafgan, HY; Abak, K. 2003. Effects of Plant Density and Number of Shoots on Yield and Fruit Characteristics of Peppers Grown in Glasshouses. Turk J. Agric. 27:29-35.

De Freitas, G; Ramos, A; Chaves, LHG; Santos Júnior, JA; Gheyi, HR. 2017. Growth and Production of Hydroponic Pepper under Salt Stress and Plant 
Density. American Journal of Plant Sciences 8(9):2255-2267.

Di Benedetto, A; Tognetti, J. 2016. Técnicas de análisis de crecimiento de plantas: su aplicación a cultivos intensivos. Ria 42(1900):258-282.

Di Rienzo, JA; Macciavelli, RE; Casanoves, F. 2012. Modelos Lineales Mixtos: aplicaciones en InfoStat. Universidad de Córdoba, Córdoba, Argentina. 193 p.

Echandi, C. 2012. Híbridos promisorios de chile dulce para la zona media-alta de Costa Rica. Boletín Técnico Quincenal de la Estación Experimental Agrícola Fabio Baudrit, Universidad de Costa Rica. Alajuela, Costa Rica. 7 p.

Elliott, J; Deryng, D; Müller, C; Frieler, K; Konzmann, M; Gerten, D; Glotter, M; Flörke, M; Wada, Y; Best, N; Eisner, S; Fekete, BM; Folberth, C; Foster, I; Gosling, SN; Haddeland, I; Khabarov, N; Ludwig, F; Masaki, Y; Olin, S; Rosenzweig, C; Ruane, AC; Satoh, Y; Schmid, E; Stacke, T; Tang, Q; Wisser, D. 2014. Constraints and potentials of future irrigation water availability on agricultural production under climate change. Proceedings of the National Academy of Sciences 111(9):3239-3244.

Fernández, MD; Gallardo, M; Bonachela, S; Orgaz, F; Thompson, RB; Fereres, E. 2005. Water use and production of a greenhouse pepper crop under optimum and limited water supply. Journal of Horticultural Science and Biotechnology 80(1):87-96.

Fernández, MD; González, AM; Carreño, J; Pérez, C; Bonachela, S. 2007. Analysis of on-farm irrigation performance in Mediterranean greenhouses. Agricultural Water Management 89:251-260.

Graham, T; Wheeler, R. 2015. Root restriction: A tool for improving volume utilization efficiency in bioregenerative life-support systems. Life Sciences in Space Research 9:62-68.

Guang-Cheng, S; Na, L; Zhan-Yu, Z; Shuang-En, Y; Changren, C. 2010. Growth, yield and water use efficiency response of greenhouse-grown hot pepper under Time-Space deficit irrigation. Scientia Horticulturae 126(2):172-179.

Hakk1, O; Yıldırım, M. 2015. Water and Radiation Use Efficiencies of Pepper (Capsicum annuum L. cv. Carliston). Sch. J. Agric. Vet. Sci. 2(2A):87-93.

Hossein, M. 2012. Effect of plant density and nitrogen fertilizer on growth, yield and fruit quality of sweet pepper (Capsicum annum L.). African Journal of Agricultural Reseearch 7(6):859-866.

Hurley, MB; Rowarth, JS; Trought, MCT; Barnes, MF; Rowe, RN. 1998. Variations in water availability and temperature in the root environment during root volume restriction studies. New Zealand Journal of Crop and Horticultural Science 26(2):127-134.

INEC (Instituto Nacional de Estadística y Censos Agropecuarios). 2015. VI Censo Nacional Agropecuario. San José, Costa Rica. 145 p.
Ismail, MR; Davies, WJ; Awad, MH. 2002. Leaf growth and stomatal sensitivity to ABA in droughted pepper plants. Scientia Horticulturae 96(1-4):313-327.

Jara, D. 2016. Evaluación de tres híbridos de pimiento (Capsicum annum L.) cultivados en hidroponía con tres mezclas de sustrato. Tesis Ing. Agr. Universidad de Guayaquil. Guayaquil, Ecuador. 46 p.

Jolliffe, PA; Gaye, MM. 1995. Dynamics of growth and yield component responses of bell peppers (Capsicum annuит L.) to row covers and population density. Scientia Horticulturae 62(3):153-164.

Karam, F; Masaad, R; Bachour, R; Rhayem, C; Rouphael, Y. 2009. Water and radiation use efficiencies in dripirrigated pepper (Capsicum annuиm L.). Response to full and deficit irrigation regimes. Europeam Journal of Horticultural Science 74(2):79-85.

Kharkina, TG; Ottosen, CO; Rosenqvist, E. 1999. Effects of root restriction on the growth and physiology of cucumber plants. Physiologia Plantarum 105(3):434-441.

Kirda, C; Topcu, S; Cetin, M; Dasgan, HY; Kaman, H; Topaloglu, F; Derici, MR; Ekici, B. 2007. Prospects of partial root zone irrigation for increasing irrigation water use efficiency of major crops in the Mediterranean region. Annals of Applied Biology 150:281-291.

Koksal, ES; Tasan, M; Artik, C; Gowda, P. 2017. Evaluation of financial efficiency of drip-irrigation of red pepper based on evapotranspiration calculated using an iterative soil water-budget approach. Scientia Horticulturae 226:398-405.

López-Marín, J; Gálvez, A; del Amor, FM; Albacete, A; Fernández, JA; Egea-Gilabert, C; Pérez-Alfocea, F. 2017. Selecting vegetative/generative/dwarfing rootstocks for improving fruit yield and quality in water stressed sweet peppers. Scientia Horticulturae 214:9-17.

López-Marín, J; González, A; Pérez-Alfocea, F; EgeaGilabert, C; Fernández, JA. 2013. Grafting is an efficient alternative to shading screens to alleviate thermal stress in greenhouse-grown sweet pepper. Scientia Horticulturae 149:39-46.

Luo, HH; Tao, XP; Hu, YY; Zhang, YL; Zhang, WF. 2015. Response of cotton root growth and yield to root restriction under various water and nitrogen regimes. Journal of Plant Nutrition and Soil Science 178(3):384-392.

Moral, R; Picó, B; Barber, X; Mininni, C; Agulló, E; Pérez-Murcia, MD; Pérez-Espinosa, A; Paredes, C; Bustamante, MA. 2014. Uso del escaneo de raíces para estimar la calidad de sustratos alternativos a la turba. In Ansorena, J; Merino, D (eds.). Actas de Horticultura, XI Jornadas de Sustratos. Barcelona, España, Sociedad Española de ciencias Hortícolas (SECH) 67:49-54. 
Moreno-Reséndez, A; Hernández-García, R; RodríguezDimas, N; Reyes-Carrillo, JL; Márquez-Quiroz, C; Preciado-Rangel, P. 2015. Development of "Serrano" pepper in vermicompost: Perlite substrates under shade net conditions. Emirates Journal of Food and Agriculture 27(12):897-902.

Nafarrate-Ramos, D; Sanchez-Soto, BH; Nunez-Ramirez, F; Lugo-Garcia, GA; Sarabia-Perea, VR; BuelnaTarin, S. 2016. Anaheim Pepper (Capsicum annuиm L.) Production on Substrates under Greenhouse Conditions. Open Access Library Journal 3(6):1-6.

NeSmith, D; Bridges, D; Barbour, J. 1992. Bell pepper responses to root restriction. Journal of Plant Nutrition 15(12):2763-2776.

Ngozi, EA; Chidera, VO. 2017. The effect of plant density on growth and yield of NsukkaYellow aromatic pepper (Capsicum annuum L.). African Journal of Agricultural Research 12(15):1269-1277.

Pérez, PJ; Castellvì, F. 2002. Análisis de la evapotranspiración a escala local y regional en cataluña. Ingeniería del agua 9(1):59-72.

Poorter, H; Bühler, J; Van Dusschoten, D; Climent, J; Postma, JA. 2012. Pot size matters: A meta-analysis of the effects of rooting volume on plant growth. Functional Plant Biology 39(11):839-850.

Qiu, R; Song, J; Du, T; Kang, S; Tong, L; Chen, R; Wu, L. 2013. Response of evapotranspiration and yield to planting density of solar greenhouse grown tomato in northwest China. Agricultural Water Management 130:44-51.

Quesada, G. 2015. Producción de chile dulce en invernadero bajo diferentes niveles de agotamiento en la humedad del sustrato. Agronomía costarricense 39(1):25-36.

Rangel, L. 2016. Crecimiento de chile habanero (Capsicum Chinense Jacq.) bajo diferentes espaciamientos entre hileras en la comarca lagunera. Tésis Lic. Coahuila, México, Universidad Autónoma Agraria Antonio Narro. 55 p.
Ritchie, JT; Basso, B. 2008. Water use efficiency is not constant when crop water supply is adequate or fixed: The role of agronomic management. European Journal of Agronomy 28(3):273-281.

Rojas, J; Paniagua, F. 2015. Comportamiento agronómico de Capsicum annuum L., Lycopersicon esculentum M. y Cucumis melo L. bajo cultivo protegido hidropónico utilizando la solución universal de Steiner. Tésis Lic. San Carlos, Costa Rica, Tecnológico de Costa Rica. 98 p.

Silva, PIB; de Negreiros, MZ; de Freitas Moura, KKC; de Freitas, FCL; de Sousa Nunes, GH; Silva, PSL e.; Grangeiro, LC. 2010. Crescimento de pimentão em diferentes arranjos espaciais. Pesquisa Agropecuaria Brasileira 45(2):132-139.

Soto, F. 2018. Parámetros para el manejo del agua en tomate y chile dulce hidropónico bajo invernadero. Agronomía Costarricense 42(2):59-73.

Taiz, L; Zeiger, E. 2006. Plant Physiology. 3 edition. Sinauer Associates, Inc. Sunderland, Massachusetts. 764 p.

Tilman, D; Cassman, KG; Matson, PA; Naylor, R; Polasky, S. 2002. Agricultural sustainability and intensive production practices. Nature 418(6898):671-677.

Vence, LB; Valenzuela, OR; Svartz, HA; Conti, ME. 2013. Elección del sustrato y manejo del riego utilizando como herramienta las curvas de retención de agua. Ciencia del Suelo 31(2):153-164.

WWAP (United Nations World Water Assessment Programme). 2016. The United Nations World Water Development Report 2016: Water and Jobs. UNESCO (Organización de las Naciones Unidas para la Educación, Ed.). Paris. 148 p.

$\mathrm{Xu}, \mathrm{G}$; Kafkafi, U. 2001. Nutrient supply and container size effects on flowering, fruiting, assimilate allocation, and water relations of sweet pepper. Acta Horticulturae 1:113-120. 